\title{
Revealing X-ray obscured quasars in SWIRE sources with extreme mid-IR/optical flux ratios
}

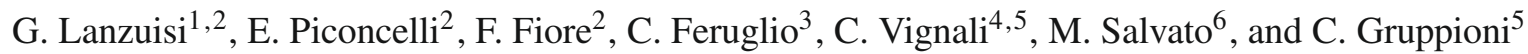 \\ 1 Dipartimento di Fisica, Università di Roma La Sapienza, P.le A. Moro 2, 00185 Roma, Italy \\ 2 Osservatorio Astronomico di Roma (INAF), via Frascati 33, 00040 Monteporzio Catone, Italy \\ e-mail: lanzuisi@oa-roma.inaf.it \\ 3 CEA, Irfu, Service d'Astrophysique, Centre de Saclay, 91191 Gif-sur-Yvette, France \\ 4 Dipartimento di Astronomia, Università degli Studi di Bologna, via Ranzani 1, 40127 Bologna, Italy \\ 5 INAF-Osservatorio Astronomico di Bologna, via Ranzani 1, 40127 Bologna, Italy \\ ${ }^{6}$ California Institute of Technology, MC 105-24, 1200 East California Boulevard, Pasadena, CA 91125, USA
}

Received 3 November 2008 / Accepted 6 February 2009

\section{ABSTRACT}

\begin{abstract}
Recent works have suggested that selection criteria based on mid-IR properties, i.e. extreme colors and bright flux levels, can be used to reveal a population of dust-enshrouded, extremely-luminous quasars at $z \sim 1-2$. However, the X-ray spectral properties of these intriguing objects still remain largely unexplored. We have performed an X-ray study of a large sample of bright mid-IR $\left(F_{24} \mu \mathrm{m}>\right.$ $1.3 \mathrm{mJy})$ galaxies showing an extreme MIR/Optical flux ratio $\left(F_{24} \mu \mathrm{m} / F_{\mathrm{R}}>2000\right)$ in order to confirm the presence of a luminous active nucleus in these very red objects. Sampling of a large area is required to pick up objects at the highest luminosities given their low surface density. Accordingly, we have applied our selection criteria to an area of $\sim 6 \mathrm{deg}^{2}$ covered by XMM-Newton/Chandra observations within the $\sim 50 \mathrm{deg}^{2}$ SWIRE survey, resulting in a final sample of 44 objects. The vast majority of the source redshifts, both spectroscopic and photometric, are in the range $0.7 \lessgtr z \lesssim 2.5$. The X-ray coverage of the sample is highly inhomogeneous (from snap-shot $5 \mathrm{ks}$ Chandra observations to medium-deep XMM-Newton exposures of $70 \mathrm{ks}$ ) and, consequently, a sizable fraction of them $(\approx 43 \%)$ remains undetected in the $0.5-10 \mathrm{keV}$ band. Using spectral or hardness information we were able to estimate the value of the absorbing column density in 23 sources. $95 \%$ of them are consistent with being obscured by neutral gas with an intrinsic column density of $N_{\mathrm{H}} \geq 10^{22} \mathrm{~cm}^{-2}$. Remarkably, we also find that $\sim 55 \%$ of these sources can be classified as type 2 quasars on the basis of their absorption properties and X-ray luminosity. Moreover, most of the X-ray undetected sources show extreme mid-IR colors, consistent with being luminous AGN-powered objects, suggesting they might host heavily obscured (possibly Compton-thick) quasars in X-rays. This demonstrates that our selection criteria applied to a wide area survey is very efficient in finding a large number of type 2 quasars at $z \gtrsim 1$. The existence of this class of very powerful, obscured quasars at high $z$ could have important implications in the context of the formation and cosmological evolution of accreting supermassive black holes and their host galaxies.
\end{abstract}

Key words. galaxies: active - galaxies: high-redshift - galaxies: nuclei - infrared: galaxies - X-rays: galaxies

\section{Introduction}

The similarity in the anti-hierarchical evolution of both star formation rate and AGN activity at $z<2$ (La Franca et al. 2005; Arnouts et al. 2007) reveals that the assembly and evolution of galaxies and those of the super massive black holes (SMBHs) at their centers are intimately connected (e.g. Page et al. 2004; Alexander et al. 2005; Hopkins et al. 2006; Best et al. 2005; Croton et al. 2006). However, the census of accreting SMBHs through different cosmic epochs is still highly incomplete and represents one of the major challenges in modern extragalactic astronomy. Clear-cut evidence of the existence of a large number of missing AGNs arises by the fact that the sources detected in the recent Chandra/XMM-Newton surveys (e.g. Brandt \& Hasinger 2005) are able to resolve only the $50-60 \%$ of the cosmic X-ray background (CXB) in the 5-10 keV band (Worsley et al. 2005), and their integrated radiation significantly fails to account for the CXB emission peak at $\sim 30 \mathrm{keV}$ (Gilli et al. 2007; Comastri 2004). A population of heavily obscured AGNs showing a spectral shape consistent with the unresolved CXB component is usually invoked to fill this gap.
Intriguingly, as stressed by Fiore et al. (2003), about $20-30 \%$ of the sources from Chandra/XMM-Newton surveys are optically-faint (i.e. $R \gtrsim 25$ ), and with a very high $\mathrm{X}$-ray-to-optical flux ratio $\left(F_{2-10} / F_{\mathrm{R}}>10\right)$. Optical spectroscopy of these extreme $F_{2-10} / F_{\mathrm{R}}$ objects is therefore extremely challenging even with the largest telescopes. Results from deep exposures and accurate multiwavelength studies indicate that the vast majority of these sources could be obscured quasars at high- $z$ (i.e. $z>1$, e.g. Mainieri et al. 2005; Maiolino et al. 2006). We have therefore started to uncover the long-sought missing AGN population of the X-ray absorbed, i.e. type 2, quasars (QSO2s hereafter), that is a key ingredient for both the CXB synthesis models as well as the prediction of the $\mathrm{SMBH} /$ galaxy formation and evolution models (e.g. Silk \& Rees 1998; Fabian 1999; Granato et al. 2004; Hopkins et al. 2006). In particular, it has been hypothesized that before shining in the optical as bright point-like blue objects, quasars undergo an early dust enshrouded phase being associated with the rapid SMBH growth. This obscured phase is likely triggered by multiple galaxy encounters and mergers that favor the infall and the concentration in the nuclear region of large reservoirs of gas feeding the SMBH as well as the assembly of the massive 
spheroids at high $z$. The link between the growth of the SMBHs and their host galaxies is believed to be strong as witnessed by the tight correlations between SMBH masses and bulge luminosity, mass and velocity dispersion (Merritt \& Ferrarese 2001). A full understanding of AGN feedback processes is therefore a key question to be addressed in the context of galaxy evolution (e.g. Di Matteo et al. 2005; Alexander et al. 2005; Menci et al. 2008; Somerville et al. 2008).

In the latest years there have been many efforts aimed at unveiling the missing population of heavily obscured quasars at high $z$ using alternative approaches with respect to the pure $\mathrm{X}$-ray selection. Multiwavelength selection criteria based on the IR band seem to be very promising, since absorbed AGN optical/UV/X-ray light should be isotropically re-emitted by the obscuring material at these frequencies. In particular, multiple independent lines of evidence point towards selection criteria using mid-IR (3-30 $\mu \mathrm{m}$; MIR hereafter) photometry or combination of MIR and multiwavelenght data, to efficiently collect large samples of optically-faint quasars candidates at $z \gtrsim 1$ (e.g. Houck et al. 2005; Yan et al. 2005; Weedman et al. 2006; Polletta et al. 2006; Alonso-Herrero et al. 2006; Daddi et al. 2007; Hickox et al. 2007; Donley et al. 2008, and references therein). Combining MIR and radio data, Martinez-Sansigre et al. (2005) claimed the discovery of a distant, optically-faint QSO2 population whose number density is comparable with that observed for classical optically bright quasars. This might explain the paucity of obscured objects in the high luminosity range of the X-ray selected AGN population that has been reported in many works (Ueda et al. 2003; Steffen et al. 2003; La Franca et al. 2005; but see also Dwelly \& Page 2006). Furthermore, recent studies have provided strong evidence that a large fraction of the dustobscured galaxies (DOGs hereafter) with extreme $F_{24} \mu \mathrm{m} / F_{\mathrm{R}}$ ratios $(\gtrsim 1000)$ and bright at $24 \mu \mathrm{m}$ (i.e. $F_{24} \mu \mathrm{m} \gtrsim 0.5-1 \mathrm{mJy}$ ), harbor a high- $z$ obscured active nucleus (e.g. Dey et al. 2008; Polletta et al. 2008a; Georgantopoluos et al. 2008; Fiore et al. 2008a,b).

However, an accurate determination of the X-ray column density of the absorber in these sources is hampered by the faintness of these sources in the X-ray band (i.e. Polletta et al. 2006; Martinez-Sansigre et al. 2007). Therefore, most of the X-ray information has been derived by the stacking of Chandra/XMMNewton data or, for small number of sources detected with only a handful of counts, by the hardness-ratio analysis.

Evidence of X-ray spectroscopically-confirmed heavilyabsorbed QSO2s at cosmological distance still remains ambiguous (i.e. Norman et al. 2002; Alexander et al. 2008; Erlund et al. 2008). Indeed, the Compton-thick (i.e. with $N_{\mathrm{H}} \gtrsim \sigma_{t}^{-1} \approx$ $1.6 \times 10^{24} \mathrm{~cm}^{-2}$ ) nature of the best Compton-thick quasar candidate, IRAS 09104+4109, has recently been questioned (and not confirmed) by Piconcelli et al. (2007a) using XMM-Newton data. One of the reasons of this difficulty is that building up large and complete samples of bright (i.e. with a $2-10 \mathrm{keV}$ flux $F_{2-10} \gtrsim$ $10^{-14} \mathrm{erg} \mathrm{cm}^{-2} \mathrm{~s}^{-1}$ ) QSO2s by homogeneous selection criteria is complex and time-consuming, because of the large area that must be covered with deep multi-band observations. This is necessary due to the low space density of the AGNs belonging to the bright end of the X-ray luminosity function. The Spitzer Wide-area InfraRed Extragalactic (SWIRE) survey (Lonsdale et al. 2003) has imaged nearly 50 square degrees in 6 fields: ELAIS-S1, ELAIS-N1, ELAIS-N2, Lockman Hole, XMM-LSS and CDFS with medium-deep MIPS and IRAC photometry, providing a unique opportunity to build up a large sample of luminous QSO2s.
In this paper we report results on an X-ray spectroscopic study of a sample of SWIRE sources selected on the basis of their extreme mid-IR/optical flux ratios $\left(F_{24} \mu \mathrm{m} / F_{\mathrm{R}}>2000\right)$ and $24 \mu \mathrm{m}$ flux density (i.e. $F_{24} \mu \mathrm{m}>1.3 \mathrm{mJy}$ ). These selection cuts have been applied in order to preferentially select sources harboring a luminous, dust-enshrouded AGN (e.g. Polletta et al. 2008a; Dey et al. 2008). Our aim is to confirm the presence of the active nucleus in these extreme DOGs and to directly measure the column density of the absorber via the characterization of their X-ray spectral properties.

The optical magnitudes used throughout the paper are Vega magnitudes. The cosmological parameters used are $H_{0}=$ $70 \mathrm{~km} \mathrm{~s}^{-1} \mathrm{Mpc}^{-1}, \Omega_{\Lambda}=0.7$ and $q_{0}=0$.

\section{The sample: selection criteria and datasets}

\subsection{Optical and IR observations}

Infrared and optical photometric data are available from the public archive of the SWIRE survey (Lonsdale et al. 2003, and references therein). The catalog contains medium-deep photometric data in MIPS $24 \mu \mathrm{m}$ band down to $400 \mu \mathrm{Jy}, 5 \sigma$, and in the four IRAC channels from 3.6 to $8.0 \mu \mathrm{m}(4.1 \mu \mathrm{Jy}$ at $3.6 \mu \mathrm{m}, 5 \sigma$, Surace et al. 2004). Optical photometry is also available in different bands for selected areas in each of the 5 SWIRE fields used in this work (ELAIS-S1, ELAIS-N1, ELAIS-N2, Lockman Hole, XMM-Newton SXDS). We did not consider the CDFS here since these data were already studied by Fiore et al. (2008a) using a $F_{24} \mu \mathrm{m} / F_{\mathrm{R}}$ selection technique similar to our approach. ELAIS-N1 and N2 have optical photometric information in 5 bands $\left(U^{\prime} g^{\prime} r^{\prime} i^{\prime} Z^{\prime}\right)$ from the Wide Field survey (WFS, McMahon et al. 2001). The Lockman Hole sky region is covered with 4-band $U g^{\prime} r^{\prime} i^{\prime}$ photometry from the SWIRE photometry program. XMM-LSS has very deep $\left(R_{A B}<27.7\right) 5$ band $B V R i^{\prime} z^{\prime}$ photometry in $1.12 \mathrm{deg}^{2}$ from the Subaru XMM Deep Survey (SXDS, e.g. Sekiguchi et al. 2005; Furusawa et al. 2008). Full details on the optical and IR photometry for the ELAIS-S1 field can be found in Feruglio et al. (2008).

We first selected sources showing a $24 \mu \mathrm{m}$ flux density $F(24 \mu \mathrm{m})>1000 \mu \mathrm{Jy}$ and $F_{24 \mu \mathrm{m}} / F_{\mathrm{R}}>1000$ in order to have an initial sample of DOGs (560 sources e.g. Fig. 1a). Several sources in ELAIS-S1, ELAIS-N1, ELAIS-N2 and Lockman Hole do not show an $R$ magnitude value in the catalogs mostly since they are faint objects, i.e. with a magnitude above the detection limit. For these sources we performed aperture photometry at the position of the MIPS source. The photometry was performed using the IRAF apphot tool, with an 1.2 arcsec aperture radius (consistent with the released optical aperture photometry), and a 10 arcsec wide annulus for background estimate. For undetected sources we computed a $3 \sigma$ limiting magnitude using the median value of $\sigma_{\mathrm{rms}}$ at the positions of the source and the relation

$\left.R_{\lim }=-2.5 \times \log (\sqrt{(} A) \times 3 \times \sigma_{\text {rms }}\right)+$ zero point

where $A$ is the area corresponding to the aperture.

\subsection{Selection criteria}

We selected all the sources with available X-ray data from the initial sample of DOGs (i.e. $F(24 \mu \mathrm{m})>1000 \mu \mathrm{Jy}$ and $F_{24 \mu \mathrm{m}} / F_{\mathrm{R}}>1000$ sources). The portion of sky covered by XMM-Newton and/or Chandra observations (for a total of $\sim 6 \mathrm{deg}^{2}$ ) significantly changes across the five SWIRE fields discussed here, varying from $\sim 100 \%$ for the XMM-SXDS field to 

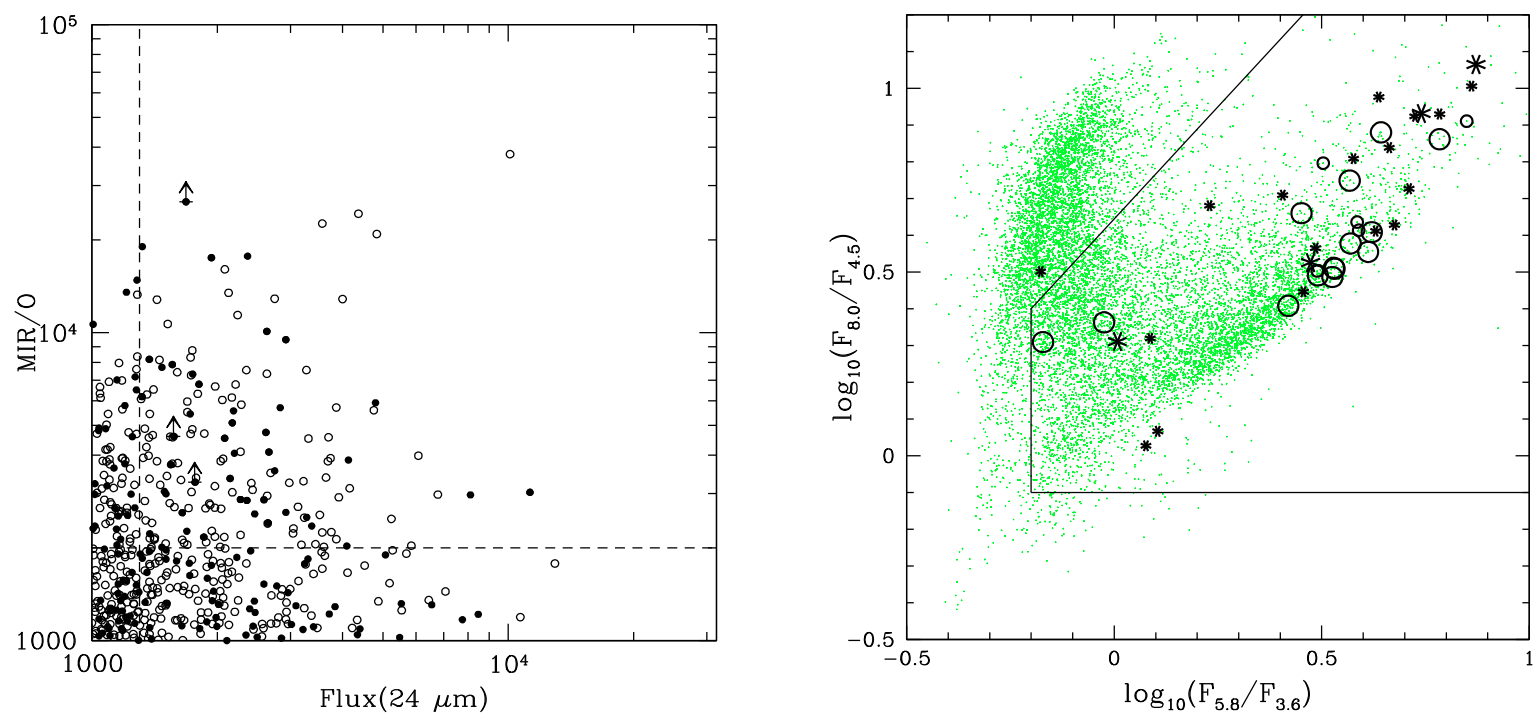

Fig. 1. a) Left panel: $24 \mu \mathrm{m}$ flux density vs. $F_{24} \mu \mathrm{m} / F_{\mathrm{R}}$ in logarithmic scales for all the sources with $F_{24 \mu \mathrm{m}} / F_{\mathrm{R}} \geq 1000$ and $F_{24 \mu \mathrm{m}} \geq 1 \mathrm{mJy}$ in the 5 SWIRE fields considered in this work. Sources with (without) available X-ray coverage are represented with filled (open) circles. Dashed lines represent the selection criteria: $F_{24 \mu \mathrm{m}} / F_{\mathrm{R}} \geq 2000$ and $24 \mu \mathrm{m}$ flux density $\geq 1.3 \mathrm{mJy}$. b) Right panel: an IRAC color-color diagram of the SWIRE $F_{24 \mu \mathrm{m}}>1 \mathrm{mJy}$ sample (small points). Sources in our sample with (without) $N_{\mathrm{H}}$ estimate are plotted with open circles (asterisks). Small (large) symbols represent sources with effective exposure time $\leq 15 \mathrm{ks}$ ( $\geq 15 \mathrm{ks}$ ). The solid line marks the region of IRAC colors typical of AGN (Lacy et al. 2004).

$\sim 5 \%$ for the ELAIS-N2 field. Nonetheless, since such a selection is completely random and not based on specific target observations, it should not introduce any selection effect in the final sample.

We started selecting sources with $F_{24} \mu \mathrm{m} / F_{\mathrm{R}}>2000$ at the highest $F_{24} \mu \mathrm{m}$ flux density levels since bright $24 \mu \mathrm{m}$ objects with large $F_{24} \mu \mathrm{m} / F_{\mathrm{R}}$ are predominantly MIR power-law (i.e. AGNdominated) sources (e.g. Dey et al. 2008; Donley et al. 2008). We then gradually decreased the $F_{24} \mu$ m flux density threshold until a statistically significant sample of objects was collected. In fact, fainter sources $\left(F_{24} \mu \mathrm{m} \sim 0.1 \mathrm{mJy}\right)$ selected with the same extreme $F_{24} \mu \mathrm{m} / F_{\mathrm{R}}$ colors, turn out to be dominated by star-formation activity both in the X-ray and MIR bands (Donley et al. 2008; Pope et al. 2008a). Accordingly we selected all the sources showing a $F_{24} \mu \mathrm{m} / F_{\mathrm{R}} \geq 2000$ and a $24 \mu \mathrm{m}$ flux density $F(24 \mu \mathrm{m}) \geq 1.3 \mathrm{mJy}$ (see Fig. 1a) obtaining in this way a sample of 50 objects, referred as Extreme DOGs (i.e. EDOGs) hereafter. Since in four cases the same optical object has a couple of IRAC-MIPS counterparts, we retained as the most likely counterpart the MIR source located at the smallest distance from the optical position.

In this way we got a sample of 46 EDOGs within the $6 \mathrm{deg}^{2}$ of SWIRE survey covered by X-ray observations. This implies a number density of $7.7 \mathrm{deg}^{-2}$ that is in very good agreement with that obtained applying the same selection criteria to the sample of DOGs in the $\approx 8.6 \mathrm{deg}^{2}$ of the NOAO Deep WideField Survey Boötes field analyzed in Dey et al. (2008) (i.e. 7.6 sources $\mathrm{deg}^{-2}$ ). The fraction of sources with $F_{24 \mu \mathrm{m}} / F_{\mathrm{R}} \geq 2000$ among all the sources with $F(24 \mu \mathrm{m}) \geq 1.3 \mathrm{mJy}$ is $\sim 10 \%$.

Two sources (namely SWIRE2 J160734.22+544217.3 and SWIRE2 J160945.43+534944.6) fall in a CCD gap in the X-ray image and therefore were excluded from the sample since there is no useful X-ray information for them. The final sample therefore consists of 44 sources and is listed in Table 1. Figure $1 \mathrm{~b}$ shows the MIR color-color diagram (e.g. Lacy et al. 2004) for the sources with $F_{24} \mu \mathrm{m}>1 \mathrm{mJy}$ in the 5 SWIRE fields considered in this work (SWIRE $F_{24} \mu \mathrm{m}>1$ sample hereafter) obtained using the flux densities measured in the four Spitzer-IRAC channels ${ }^{1}$. Two distinct populations can be readily separated in this plot. One has blue colors in $F_{5.8} / F_{3.6}$ and red colors in $F_{8.0} / F_{4.5}$, most likely consisting of a mixture of starlight-dominated galaxies and star-forming galaxies with MIR 5-12 $\mu \mathrm{m}$ spectra dominated by PAH emission features at low-redshift $(z \lesssim 0.2-0.4)$. The other population has redder colors and can be mainly identified with galaxies with a MIR power-law spectrum that is a typical signature of an AGN-powered emission. The thick lines mark the region of the diagram typically occupied by AGNs (Lacy et al. 2004; Alonso-Herrero et al. 2006; Polletta et al. 2006; Lacy et al. 2007; Barmby et al. 2008; see also Donley et al. 2008, for a detailed discussion about the level of contamination by starforming galaxies in this region). It is worth noting that all but one sources in our sample lie within the AGN region, and most of them show extreme red colors i.e. $\log \left(F_{5.8} / F_{3.6}\right) \gtrsim 0.4$ and $\log \left(F_{8.0} / F_{4.5}\right) \gtrsim 0.4$ similarly to the luminous obscured quasars at $z=1.3-3$ in the Polletta et al. (2008a) sample. The only source falling outside the box has the lowest spectroscopic redshift in the sample, i.e. source No. 14 at $z=0.22$, and therefore it is likely that the contribution from the host galaxy stellar light can explain the low value of the $F_{5.8} / F_{3.6}$ ratio. The range of MIR colors observed for our EDOGs can be likely ascribed to the relative strength of the contribution of star formation to the total MIR luminosity (e.g. Dey et al. 2008; Sajina et al. 2005). Interestingly, Donley et al. (2007) and Cardamone et al. (2008) found that the most luminous X-ray sources with $L_{2-10} \gtrsim 5 \times 10^{44} \mathrm{erg} \mathrm{s}^{-1}$ in their large samples of MIR sources fall in this box of the IRAC color-color diagram.

\subsection{Redshifts}

Spectroscopic redshifts are available for 7 objects in the sample $\left(0.22 \leq z_{\text {sp }} \leq 2.54\right.$; e.g. Table 1$)$. In particular, two of them are

\footnotetext{
1 Source Nos. 8, 9, 25 and 43 are undetected in one (or two) IRAC bands and, therefore, they are not plotted in Fig. $1 \mathrm{~b}$.
} 
Table 1. Optical-IR parameters and redshift of the sample.

\begin{tabular}{|c|c|c|c|c|c|c|c|c|c|c|c|}
\hline $\begin{array}{l}\text { ID } \\
\text { (1) }\end{array}$ & $\begin{array}{l}\text { Name } \\
(2)\end{array}$ & $\begin{array}{l}R \\
(3)\end{array}$ & $\begin{array}{c}F_{3.6 \mu \mathrm{m}} \\
(4)\end{array}$ & $\begin{array}{c}F_{4.5 \mu \mathrm{m}} \\
(5)\end{array}$ & $\begin{array}{l}F_{5.8 \mu \mathrm{m}} \\
(6)\end{array}$ & $\begin{array}{l}F_{8 \mu \mathrm{m}} \\
(7)\end{array}$ & $\begin{array}{c}F_{24 \mu \mathrm{m}} \\
(8)\end{array}$ & $\begin{array}{c}\log \left(F_{24 \mu \mathrm{m}} / F_{\mathrm{R}}\right) \\
(9)\end{array}$ & $\begin{array}{c}\log \left(\lambda L_{5.8 \mu \mathrm{m}}\right) \\
(10)\end{array}$ & $\begin{array}{c}z \\
(11)\end{array}$ & $\begin{array}{l}z \text {-type } \\
(12)\end{array}$ \\
\hline 1 & J021559.46-045517.5 & 25.65 & 148.1 & 248.8 & 389.1 & 636.6 & 2364 & 4,2 & 45.14 & 1.007 & $\mathrm{SP}^{a}$ \\
\hline 2 & J021625.91-050425.1 & 24.39 & 14.31 & 34.45 & 106.5 & 400.0 & 2174 & 3.7 & 45.96 & 2.236 & $\mathrm{PH}^{h}$ \\
\hline 3 & J021656.59-051005.3 & 24.31 & 83.82 & 146.1 & 249.2 & 490.0 & 2083 & 3.7 & 45.12 & 1.080 & $\mathrm{PH}^{h}$ \\
\hline 4 & J021734.65-051718.2 & 26.36 & 59.84 & 111.0 & 222.1 & 419.7 & 1322 & 4.3 & 45.64 & 1.938 & $\mathrm{PH}^{h}$ \\
\hline 5 & J021743.09-044921.6 & 24.20 & 146 & 270.2 & 496.0 & 871.9 & 1508 & 3.5 & 45.13 & 1.042 & $\mathrm{PH}^{h}$ \\
\hline 6 & J021749.00-052306.9 & 22.36 & 375.6 & 714.2 & 1271 & 2317 & 8105 & 3.5 & 45.67 & 0.914 & $\mathrm{SP}^{a}$ \\
\hline 7 & J021754.45-043016.0 & 23.34 & 132.5 & 243.9 & 509.9 & 1055 & 2922 & 3.4 & 45.91 & 1.780 & $\mathrm{PH}^{h}$ \\
\hline 8 & J021912.66-043308.5 & 23.80 & 27.39 & 21.36 & - & - & 1471 & 3.3 & 44.42 & 0.754 & $\mathrm{PH}^{h}$ \\
\hline 9 & J021924.57-045300.0 & 25.26 & 30.38 & 42.14 & 67.16 & - & 1473 & 3.9 & 45.62 & 1.979 & $\mathrm{PH}^{h}$ \\
\hline 10 & J021935.19-050528.4 & 22.70 & 159.8 & 142.5 & 151.1 & 328.6 & 4095 & 3.3 & 44.76 & 0.826 & $\mathrm{SP}^{b}$ \\
\hline 11 & J022003.58-045145.6 & 23.07 & 123 & 215.7 & 380.7 & 667.7 & 3374 & 3.4 & 45.28 & 1.080 & $\mathrm{PH}^{h}$ \\
\hline 12 & J022003.95-045220.4 & 24.22 & 48.1 & 77.87 & 177.6 & 436.7 & 2831 & 3.7 & 45.50 & 1.443 & $\mathrm{PH}^{h}$ \\
\hline 13 & $\mathrm{~J} 160623.44+542301.9$ & 24.59 & 35.85 & 41.66 & 43.79 & 86.93 & 1546 & 3.6 & 45.05 & 1.466 & $\mathrm{PH}^{h}$ \\
\hline 14 & $\mathrm{~J} 160650.71+542510.1$ & $>24.30$ & 290.2 & 258.2 & 193.1 & 818.8 & 1768 & $>3.5$ & 43.66 & 0.22 & $\mathrm{SP}^{c}$ \\
\hline 15 & $\mathrm{~J} 160700.62+542416.8$ & 26.02 & 18.04 & 37.57 & 95.66 & 315.3 & 1937 & 4.2 & 44.35 & $0.63_{-0.20}^{+0.20}$ & $\mathrm{PH}^{g}$ \\
\hline 16 & $\mathrm{~J} 160813.86+541942.4$ & 25.09 & 63.38 & 130.0 & 270.4 & 531.2 & 2631 & 4.0 & 45.09 & 1 & $\mathrm{PH}^{g}$ \\
\hline 17 & $\mathrm{~J} 160816.15+545351.1$ & 24.12 & 64.9 & 127.7 & 333.1 & 680.6 & 2147 & 3.5 & 44.75 & $0.70_{-0.15}^{+0.15}$ & $\mathrm{PH}^{g}$ \\
\hline 18 & $\mathrm{~J} 160913.02+540509.7$ & 25.31 & 61.84 & 136.9 & 292.7 & 581.3 & 1319 & 3.8 & 45.02 & 1 & $\mathrm{PH}^{g}$ \\
\hline 19 & $\mathrm{~J} 160913.28+542322.0$ & 23.86 & 254.4 & 478.6 & 987.4 & 1966 & 4798 & 3.8 & 45.90 & 1.400 & $\mathrm{PH}^{h}$ \\
\hline 20 & $\mathrm{~J} 160933.78+535720.2$ & 25.57 & 50.39 & 69.80 & 60.08 & 74.27 & 1373 & 3.9 & 44.43 & 1 & $\mathrm{PH}^{g}$ \\
\hline 21 & $\mathrm{~J} 160950.98+535836.3$ & 23.95 & 131.9 & 236.4 & 406.9 & 752.3 & 1692 & 3.3 & 45.26 & 1.138 & $\mathrm{PH}^{h}$ \\
\hline 22 & $\mathrm{~J} 161007.17+540628.5$ & 23.34 & 38.47 & 90.59 & 271.9 & 737.3 & 3284 & 3.4 & 45.95 & 1.871 & $\mathrm{PH}^{h}$ \\
\hline 23 & $\mathrm{~J} 161017.70+535124.1$ & $>24.80$ & 17.48 & 37.81 & 80.36 & 260.6 & 1572 & $>3.7$ & 44.37 & $0.69_{-0.20}^{+0.20}$ & $\mathrm{PH}^{g}$ \\
\hline 24 & $\mathrm{~J} 161017.93+542332.1$ & 23.89 & 36 & 58.41 & 135.9 & 375.9 & 2273 & 3.5 & 44.42 & $0.63_{-0.10}^{+0.10}$ & $\mathrm{PH}^{g}$ \\
\hline 25 & $\mathrm{~J} 161037.63+540603.5$ & 24.88 & 12.05 & 12.63 & - & 50.06 & 1724 & 3.7 & 46.58 & $3.77_{-0.77}^{+0.48}$ & $\mathrm{PH}^{g}$ \\
\hline 26 & $\mathrm{~J} 161051.03+535330.5$ & 25.39 & 13.26 & 20.86 & 57.56 & 197.4 & 1560 & 3.9 & 44.22 & $0.66_{-0.15}^{+0.15}$ & $\mathrm{PH}^{g}$ \\
\hline 27 & $\mathrm{~J} 161052.74+543740.4$ & 23.53 & 70.77 & 66.55 & 72.03 & 136.1 & 2646 & 3.4 & 45.21 & 1.410 & $\mathrm{PH}^{h}$ \\
\hline 28 & $\mathrm{~J} 161102.22+550551.6$ & 24.10 & 71.53 & 73.24 & 121.4 & 350.9 & 2661 & 3.6 & 44.97 & 0.995 & $\mathrm{PH}^{h}$ \\
\hline 29 & J161204.84+544149.2 & 25.07 & 33.74 & 46.92 & 42.97 & 54.68 & 1808 & 3.8 & 45.98 & $2.58_{-0.30}^{+0.40}$ & $\mathrm{PH}^{g}$ \\
\hline 30 & $\mathrm{~J} 161306.28+543959.7$ & 24.91 & 23.5 & 54.15 & 170.7 & 548.9 & 2922 & 4.0 & 44.40 & $0.54_{-0.10}^{+0.10}$ & $\mathrm{PH}^{g}$ \\
\hline 31 & $\mathrm{~J} 163723.94+410525.7$ & 23.80 & 77.52 & 65.69 & 52.22 & 133.9 & 1856 & 3.3 & 44.94 & 1.24 & $\mathrm{PH}^{h}$ \\
\hline 32 & $\mathrm{~J} 104409.95+585224.7$ & 23.56 & 64.27 & 150.4 & 390.9 & 1093 & 4134 & 3.6 & 46.41 & 2.54 & $\mathrm{SP}^{d}$ \\
\hline 33 & $\mathrm{~J} 104528.29+591326.6$ & 23.68 & 32.18 & 45.19 & 90.94 & 206.6 & 2462 & 3.4 & 45.98 & 2.31 & $\mathrm{SP}^{e}$ \\
\hline 34 & $\mathrm{~J} 104837.82+572816.2$ & 23.84 & 100.6 & 170.3 & 307.5 & 625.3 & 2355 & 3.5 & 45.53 & 1.443 & $\mathrm{PH}^{h}$ \\
\hline 35 & J104847.14+572337.6 & 23.74 & 158.1 & 285.4 & 451.8 & 797.1 & 2590 & 3.5 & 45.42 & 1.218 & $\mathrm{PH}^{h}$ \\
\hline 36 & $\mathrm{~J} 104954.96+584429.4$ & 24.15 & 18.45 & 23.43 & 58.82 & 146.6 & 1378 & 3.3 & 44.27 & $0.76_{-0.10}^{+0.10}$ & $\mathrm{PH}^{g}$ \\
\hline 37 & $\mathrm{~J} 105604.84+574229.9$ & 22.21 & 62.28 & 85.63 & 158.6 & 438.5 & 11280 & 3.5 & 45.59 & 1.249 & $\mathrm{PH}^{h}$ \\
\hline 38 & J003314.61-432300.3 & 24.13 & 10.5 & 16.69 & 57.68 & 142.5 & 1648 & 3.4 & 44.46 & $0.87_{-0.20}^{+0.20}$ & $\mathrm{PH}^{g}$ \\
\hline 39 & J003316.92-431706.3 & 24.30 & 142.2 & 295.1 & 581.9 & 1059 & 2197 & 3.6 & 44.88 & 0.689 & $\mathrm{SP}^{f}$ \\
\hline 40 & J003333.75-432326.9 & 24.41 & 48.02 & 73.56 & 161.1 & 225.4 & 1493 & 3.5 & 45.03 & $1.24_{-0.20}^{+0.20}$ & $\mathrm{PH}^{g}$ \\
\hline 41 & J003336.26-431731.7 & 24.65 & 18.66 & 34.14 & 82.01 & 259.0 & 2188 & 3.7 & 44.51 & $0.76_{-0.10}^{+0.10}$ & $\mathrm{PH}^{g}$ \\
\hline 42 & J003348.18-432822.3 & $>26.63$ & 16.17 & 40.72 & 98.36 & 346.5 & 1682 & $>4.4$ & 44.70 & $0.84_{-0.05}^{+0.05}$ & $\mathrm{PH}^{g}$ \\
\hline 43 & J003518.20-433414.6 & 25.19 & 12.06 & 16.96 & - & 142.6 & 1745 & 3.9 & 45.38 & $1.69_{-1.00}^{+0.45}$ & $\mathrm{PH}^{g}$ \\
\hline 44 & J003641.47-432038.1 & 23.91 & 110 & 224.6 & 459.3 & 911.7 & 2746 & 3.6 & 45.25 & 1 & $\mathrm{PH}^{g}$ \\
\hline
\end{tabular}

Column: (1) ID number; (2) name from SWIRE2 catalogue; (3) magnitude $R$; (4) $3.6 \mu \mathrm{m}$ flux density in $\mu \mathrm{Jy}$; (5) $4.5 \mu \mathrm{m}$ flux density in $\mu \mathrm{Jy}$; (6) $5.8 \mu \mathrm{m}$ flux density in $\mu \mathrm{Jy}$; (7) $8 \mu \mathrm{m}$ flux density in $\mu \mathrm{Jy}$; (8) $24 \mu \mathrm{m}$ flux density in $\mu \mathrm{Jy}$; (9) logarithm of $F_{24} \mu \mathrm{m} / F_{\mathrm{R}}$ flux ratio; (10) logarithm of $5.8 \mu \mathrm{m}$ luminosity in $\mathrm{erg} \mathrm{s}^{-1}$; (11) redshift; (12) type of redshift: SP spectroscopic redshift [ ${ }^{a}$ this work (see Sect. 2.3 .1 for details); ${ }^{b}$ Simpson et al. (2006); ${ }^{c}$ from Sloan Digital Sky Survey Data Release $2 ;{ }^{d}$ Polletta et al. (2006); ${ }^{e}$ Weedman et al. (2006a); ${ }^{f}$ Feruglio et al. (2008)], PH photometric redshift $\left[{ }^{g}\right.$ this work (see Sect. 2.3 for details); ${ }^{h}$ Rowan-Robinson et al. (2008); ${ }^{i}$ fixed value of $\left.z\right]$.

obtained from new observations performed with the Subaru telescope (see Sect. 2.3.1 for details of data reduction and analysis).

For the remaining 37 sources we were able to derive photometric redshifts thanks to the available optical and IR photometric data. We used the code described in Fontana et al. (2000) and Fontana (2001). Photometric redshifts were calculated using a $\chi^{2}$ minimization technique on spectral libraries which include starburst, passive galaxy and AGN semi-empirical template spectra from Polletta et al. (2007), Fiore et al. (2008a) and Pozzi at al. (2007). All the available photometric data points, from the optical to the MIPS $24 \mu \mathrm{m}$ band, were used in the computation (e.g. Feruglio et al. 2008). The photo- $z$ are reported in Table 1 with errors corresponding to a confidence level of $1.6 \sigma$ and their distribution spans in the range $0.54 \leq z_{\mathrm{ph}} \leq 3.77$.

In four cases the $\chi^{2}$ minimization technique was not able to properly constrain the $z$ value. For these sources (namely 

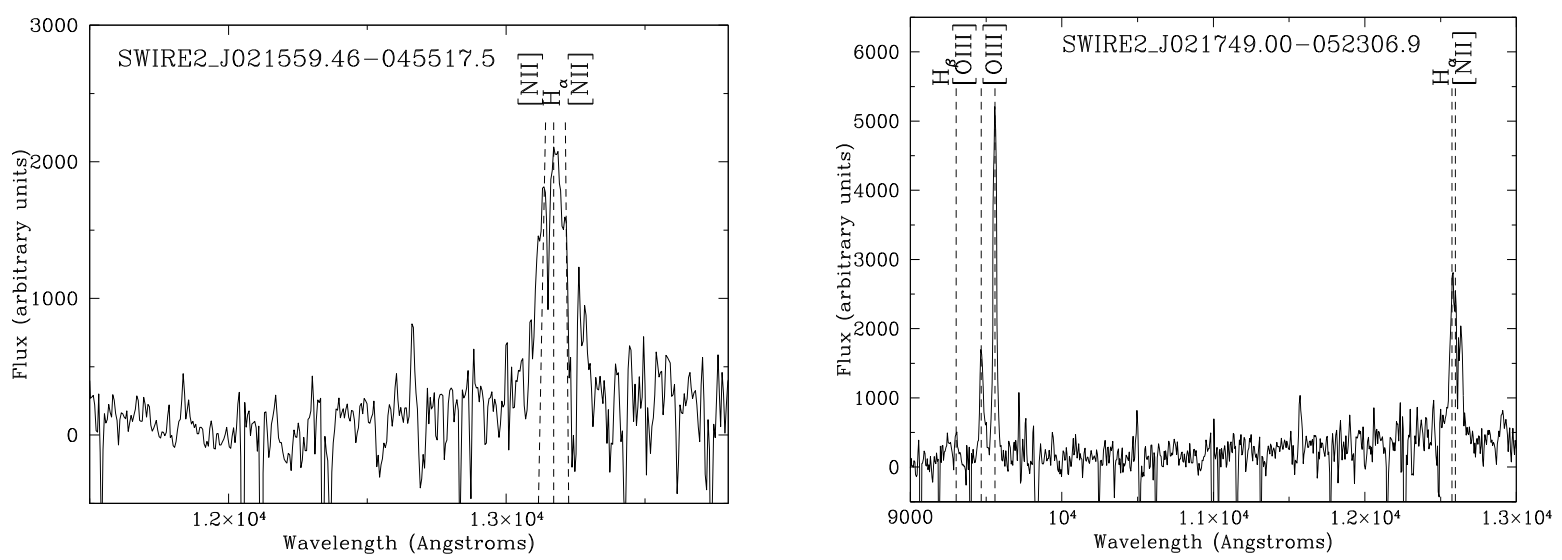

Fig. 2. a) Left panel: near IR spectrum of source No. 1 at $z=1.007$. b) Right panel: near IR spectrum of source No. 6 at $z=0.914$. Both spectra were obtained by MOIRCS/SUBARU observations.

Nos. 16, 18, 20 and 44) we decided to assume a fixed value of $z=1$ which is close to the mean value of the $z$ distribution in our sample $(\langle z\rangle \sim 1.2)$. It is worth noting that this is a conservative assumption since the typical value of $z$ found for large samples of DOGs is 2 (e.g. Polletta et al. 2008a; Dey et al. 2008, and references therein).

For 19 of 37 sources photometric redshifts from the SWIRE Photometric Redshift Catalogue published in Rowan-Robinson et al. (2008) are available ${ }^{2}$. Their approach is based on galaxy and quasar templates applied to data at $0.36-4.5 \mu \mathrm{m}$, and on a set of 4 IR emission templates fitted to IR excess data at 3.6$170 \mu \mathrm{m}$. The photo- $z$ distribution from the SWIRE Photometric Redshift Catalogue spans in the range $0.754 \leq z_{\text {ph }} \leq 2.236$.

\subsubsection{Spectroscopic redshifts}

We obtained the spectroscopic redshifts for two sources (namely source Nos. 1 and 6) via near-IR spectroscopy. These observations were performed with MOIRCS (Multi-Object InfraRed Camera and Spectrograph) mounted on the Subaru telescope (Hawaii) on 2007 Dec. 22, as backup program (program No. S07B0087N, P.I. M. Salvato). We used the zJ500 grism (optimized for the observation of $0.9-1.78 \mu$ m region) with a $\mathrm{R} 500$ filter blocking the contamination of the second-order spectra at $\lambda>1.5 \mu \mathrm{m}$. Each observation consists of 2 frames dithered along the slit by 5 arcsec.

After standard reduction the spectra have been subtracted by each other in order to improve sky subtraction and flatfielding and then combined. SWIRE2 J021559.46-045517.5 has been observed for a total of $1200 \mathrm{~s}$ and SWIRE2 J021749.00052306.9 for a total of $1800 \mathrm{~s}$. Sky lines have been used for the wavelength calibration. Results can be summarized as follows:

- SWIRE2 J021559.46-045517.5 (No. 1): we obtained a redshift of $z=1.007$ on the basis of the identification of $\mathrm{H}_{\alpha} \lambda 6563\left(F W H M<1100 \mathrm{~km} \mathrm{~s}^{-1}\right)[\mathrm{NII}] \lambda 6548$ and [NII] $\lambda 6583$ narrow emission lines (see Fig. 2a).

- SWIRE2 J021749.00-052306.9 (No. 6): we obtained a redshift of $z=0.914$ on the basis of the identification of strong [OIII] $\lambda 49959, \lambda 5007, \mathrm{H}_{\alpha} \lambda 6563$ and [NII] $\lambda 6583$ narrow emission lines (see Fig. 2b). This value does not match

2 For 15 sources we were able to compare the photo- $z$ from the SWIRE Photometric Redshift Catalogue with those computed with our method. We found that for $\sim 50 \%(\sim 85 \%)$ of the sources, the photo- $z$ values are consistent within $15 \%(30 \%)$. with the tentative redshift of $z=0.987$ proposed by Lacy et al. (2007), using very low $\mathrm{S} / \mathrm{N}$ optical spectral data. The $F W H M$ of the $\mathrm{H}_{\alpha}$ line is $F W H M<1000 \mathrm{~km} \mathrm{~s}^{-1}$. This support a narrow line quasar classification for this $E D O G$.

\subsection{MIR properties of EDOGs}

We computed $5.8 \mu \mathrm{m}$ monochromatic luminosities of our sources using a linear interpolation between the observed $8 \mu \mathrm{m}$ and $24 \mu \mathrm{m}$ flux densities at the wavelength corresponding to 5.8 $\mu \mathrm{m}$ at the source rest-frame (e.g. Fiore et al. 2008a, for further details). The $F_{24} \mu \mathrm{m} / F_{\mathrm{R}}$ values as a function of the $\lambda L_{5.8 \mu \mathrm{m} \mathrm{lu}}$ minosity for the SWIRE $F_{24} \mu \mathrm{m}>1 \mathrm{mJy}$ sample and for our EDOG sample are compared in Fig. $3 \mathrm{a}$.

It can be seen that our selection criteria $\left(F_{24} \mu \mathrm{m} / F_{\mathrm{R}}>2000\right.$ and $F_{24} \mu \mathrm{m}>1.3 \mathrm{mJy}$ ) are well suited to find highly obscured, high-luminosity $\left(\lambda L_{5.8 \mu \mathrm{m}} \geq 10^{44} \mathrm{erg} \mathrm{s}^{-1}\right)$ sources. Furthermore, the combination of large $F_{24} \mu \mathrm{m}$ and extreme MIR colors guarantees that the vast majority of our EDOGs are AGN-dominated in the MIR band (e.g. Polletta et al. 2008a; Dey et al. 2008; Donley et al. 2008). Our finding is therefore in agreement with the correlation between $F_{24 \mu \mathrm{m}} / F_{\mathrm{R}}$ and MIR luminosity reported by Fiore et al. (2008a) for obscured AGNs (see their Figs. 2a and b).

On the other hand, highly luminous (i.e. $\lambda L_{5.8 \mu \mathrm{m}}>$ $10^{44} \mathrm{erg} \mathrm{s}^{-1}$ ) MIR sources with a $F_{24 \mu \mathrm{m}} / F_{\mathrm{R}}$ value in the range 10-100 predominantly show a continuum-dominated MIR spectrum with PAH emission features, suggesting they are composite ULIRG systems containing both a dominant AGN and a starburst component (Sajina et al. 2007; Yan et al. 2007). MIRselected, luminous type 1 quasars typically fall in this region of the $F_{24 \mu \mathrm{m}} / F_{\mathrm{R}}-\lambda L_{5.8} \mu \mathrm{m}$ diagram (e.g. Brand et al. 2007; Fiore et al. 2008b; Polletta et al. 2008a). Finally, sources at lower luminosities (i.e. $\lambda L_{5.8 \mu \mathrm{m}}<10^{44} \mathrm{erg} \mathrm{s}^{-1}$ ) can be likely associated with Seyfert-like AGNs (obscured and unobscured) and starburst galaxies.

In Fig. $3 \mathrm{~b}$ is shown the distribution of the $\lambda L_{5.8 \mu \mathrm{m}}$ luminosities as a function of the redshift for our EDOGs sample. For comparison we also plot the sources from the CDFS and COSMOS fields (Fiore et al. 2008a,b) with $F_{24} \mu \mathrm{m} / F_{\mathrm{R}}>2000$. The combination of our selection criteria with the large area of the SWIRE survey allows us to collect the most IR luminous sources at all redshifts: objects in our EDOG sample are, on average, $\gtrsim 0.5(\gtrsim 1.5-2)$ order of magnitude brighter than those selected in the $2(<0.1) \mathrm{deg}^{2}$ COSMOS (CDFS) survey. Assuming that EDOGs host an AGN at their center, it is possible to give a 

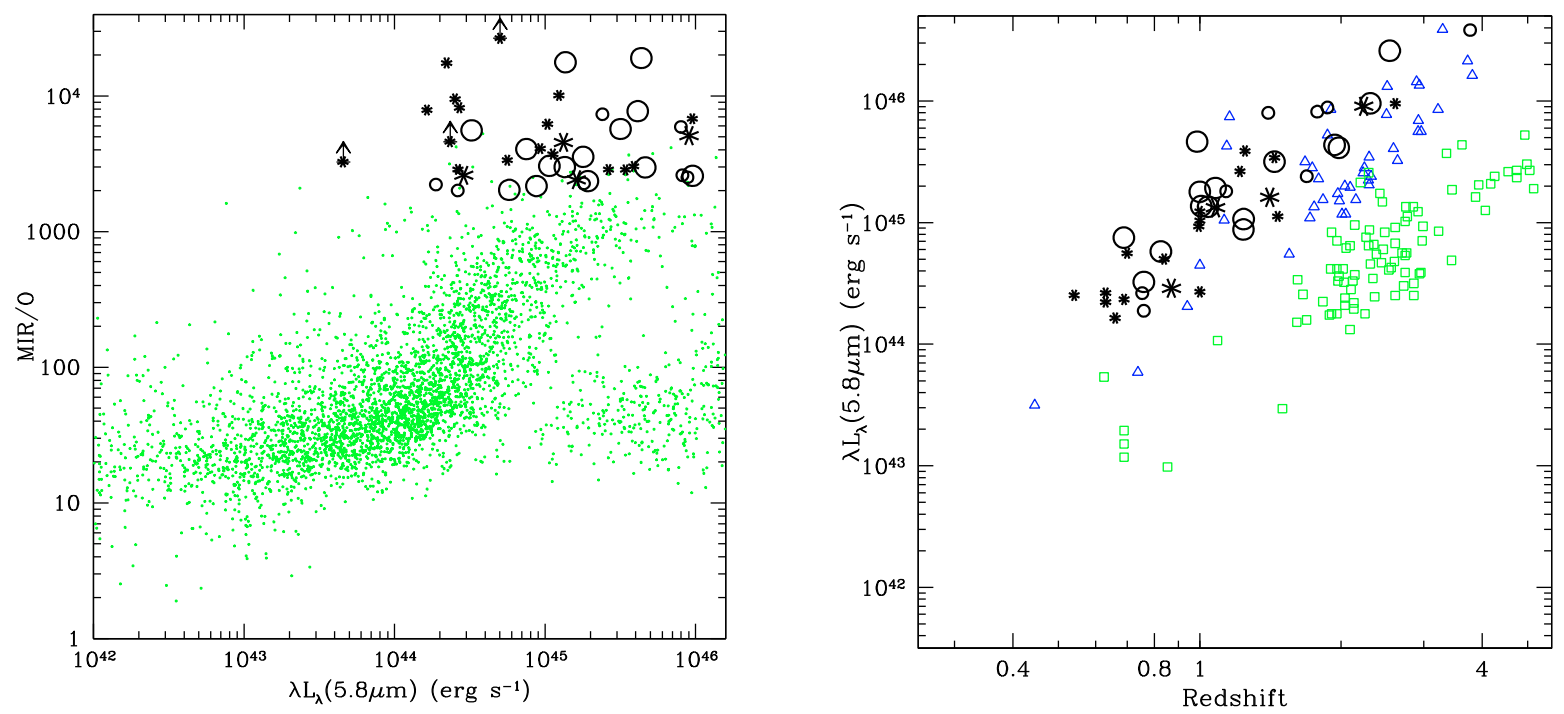

Fig. 3. a) Left panel: $\lambda L_{5.8 \mu \mathrm{m}}$ vs. $F_{24 \mu \mathrm{m}} / F_{\mathrm{R}}$ ratio for the SWIRE $F_{24 \mu \mathrm{m}}>1 \mathrm{mJy}$ sample (small points). Sources in our sample with (without) $N_{\mathrm{H}}$ estimate are plotted with open circles (asterisks). Small (large) symbols represent sources with effective exposure time $\leq 15 \mathrm{ks}(\geq 15 \mathrm{ks})$. b) Right panel: the $\lambda L_{5.8 \mu \mathrm{m}}$ - redshift plane for sources in our sample (symbol as in the left panel), COSMOS sources (open triangles) and CDFS sources (open squares) with $F_{24} \mu \mathrm{m} / F_{\mathrm{R}}>2000$ from Fiore et al. (2008a,b).

rough estimate of the bolometric luminosity $\left(L_{\mathrm{Bol}}\right)$ using the relationship $\log L_{\mathrm{Bol}}=\log \lambda L_{5.8} \mu \mathrm{m}+1.16$ from Elvis et al. (1994). In particular, Polletta et al. (2008a) point out that this relationship likely provides only a lower limit on real value of $L_{\mathrm{Bol}}$. By proceeding in this way we obtained values of bolometric luminosities in the range $10^{12} \lesssim L_{\mathrm{Bol}} \lesssim 10^{14} L_{\odot}$. EDOGs are therefore placed at the brightest end of the bolometric luminosity function of galaxies in the Universe.

\section{X-ray observations}

\subsection{Data reduction}

We searched for all Chandra and XMM-Newton archival observations available for the SWIRE fields listed in Sect. 2.1 as of February 2008. The X-ray coverage of the sources in our sample is highly inhomogeneous, ranging from shallow 5 ks Chandra observations of the Boötes/NOAO field, up to medium-deep observations in the XMM-Newton SXDS field $(\sim 50 \mathrm{ks})$ and in the Chandra Lockman Hole region $(\sim 70 \mathrm{ks})$. Twenty-one sources fall within the FOV of an XMM-Newton observation, while the sources with Chandra data are twenty-three.

Event files of the Chandra observations were retrieved from the Chandra X-ray Center (CXC) via the Web ChaSeR (Chandra Archive Search and Retrieval) interface. The data reduction was performed following the standard procedures outlined in the Science Analysis Threads for ACIS data at the CIAO Web site ${ }^{3}$.

Standard XMM-Newton SAS tasks epproc and emproc (SAS v. 7.1.0 $)^{4}$ were used to linearize the $P N$ and $M O S$ event files. The event files were processed using the latest calibration files and cleaned up from effects of hot pixels and cosmic rays contamination. X-ray events corresponding to patterns 0-12 (0-4) for the $\operatorname{MOS}(P N)$ cameras were selected. XMM-Newton observations sometimes experience high background flaring periods due to the crossing of clouds of soft (i.e. $E<1 \mathrm{MeV}$ ) protons, produced by the sun and projected towards the Earth in the solar wind, that are accelerated by magnetic reconnections in the

\footnotetext{
3 http://cxc.harvard.edu/ciao

4 http://xmm. esac.esa.int/sas/
}

magnetosphere. In order to produce a cleaned event file we removed all the high background periods identified in light curves at energies $>10 \mathrm{keV}$, where the contribution from the emission from real X-ray sources is minimized.

\subsection{X-ray fluxes}

The count-rate in the $0.5-10 \mathrm{keV}$ band (indicated as $\mathrm{T}$ band hereafter) was obtained by the command sosta in the Ximage ${ }^{5}$ package.

Using this command, we derived the number of source counts within a box region with a 5 (15) arcsec side for Chandra (XMM-Newton) data centered on the position of the optical counterpart of the X-ray source and also corrected them for the effects due to the mirror vignetting and the off-axis point spread function degradation. We repeated the same procedure to estimate the background by the extraction of the background counts from a source-free region around the target. We were thus able to infer the background-subtracted source count rate and its statistical significance. A $3 \sigma$ upper limit is automatically calculated by sosta if the detection significance is less than $10^{-3}$. According to this procedure, we obtained 25 positive detections and 19 upper limits in the $\mathrm{T}$ band for the 44 sources in our sample.

The count rate was subsequently converted into flux using the PIMMS ${ }^{6}$ tool. As underlying spectral model we assumed a power law (with a photon index fixed to $\Gamma=1.9$ ) modified by absorption from neutral gas with a rest-frame column density of $N_{\mathrm{H}}=10^{23} \mathrm{~cm}^{-2}$. This is an intermediate $N_{\mathrm{H}}$ value for $F_{24 \mu \mathrm{m}} / F_{\mathrm{R}}$ selected DOGs (see below).

Table 2 lists the resulting $\mathrm{T}$ band count rate and flux along with the effective exposure time for each source in the sample. The latter has been obtained from the nominal exposure time of the observation after correction for the vignetting factor at the source position on the detector. We found that $\sim 45 \%$ of the sources have an effective exposure time that is $\geq 15 \mathrm{ks}$. Fluxes in

\footnotetext{
5 http://heasarc.gsfc.nasa.gov/xanadu/ximage/ximage. html

${ }^{6}$ http://heasarc . nasa.gov/Tools/w3pimms.html
} 
Table 2. X-ray coverage and $0.5-10 \mathrm{keV}$ band flux of the sample.

\begin{tabular}{|c|c|c|c|c|c|}
\hline $\begin{array}{l}\text { ID } \\
\text { (1) }\end{array}$ & $\begin{array}{l}\text { Exposure } \\
\text { (2) }\end{array}$ & $\begin{array}{c}\text { Telescope } \\
\text { (3) }\end{array}$ & $\begin{array}{c}\mathrm{CR}_{0.5-10} \\
\text { (4) }\end{array}$ & $\begin{array}{c}\text { Flux }_{0.5-10} \\
(5)\end{array}$ & $\begin{array}{c}\log \left(F_{2-10} / F_{\mathrm{R}}\right) \\
(6)\end{array}$ \\
\hline 1 & 18.8 & $X M M-N$ & $25.6 \pm 4.3$ & $18.2 \pm 3.1$ & $50.1 \pm 8.4$ \\
\hline 2 & 23.2 & $X M M-N$ & $2.1 \pm 0.9$ & $1.8 \pm 0.8$ & $<2.0$ \\
\hline 3 & 29.3 & $X M M-N$ & $<2.4$ & $<2.1$ & $<2.3$ \\
\hline 4 & 38.0 & $X M M-N$ & $3.9 \pm 1.2$ & $9.4 \pm 5.1$ & $79.4 \pm 24.4$ \\
\hline 5 & 31.8 & $X M M-N$ & $3.5 \pm 1.8$ & $2.4 \pm 1.1$ & $0.4 \pm 0.2$ \\
\hline 6 & 32.1 & $X M M-N$ & $86.9 \pm 5.1$ & $111 \pm 5.5$ & $131.8 \pm 7.7$ \\
\hline 7 & 6.8 & $X M M-N$ & $11.5 \pm 2.0$ & $15.6 \pm 2.7$ & $8.1 \pm 3.4$ \\
\hline 8 & 9.0 & $X M M-N$ & $3.2 \pm 1.6$ & $3.7 \pm 1.6$ & $3.0 \pm 1.5$ \\
\hline 9 & 21.7 & $X M M-N$ & $4.7 \pm 1.9$ & $6.0 \pm 2.3$ & $18.2 \pm 9.7$ \\
\hline 10 & 23.7 & $X M M-N$ & $2.1 \pm 1.5$ & $2.6 \pm 1.6$ & $<0.7$ \\
\hline 11 & 17.0 & $X M M-N$ & $8.5 \pm 3.3$ & $6.8 \pm 2.3$ & $2.6 \pm 1.0$ \\
\hline 12 & 17.6 & $X M M-N$ & $17.6 \pm 3.0$ & $29.1 \pm 9.0$ & $37.2 \pm 14.7$ \\
\hline 13 & 4.7 & Chandra & $<13.6$ & $<16.3$ & $<22.9$ \\
\hline 14 & 4.4 & Chandra & $<23.7$ & $<41.9$ & $<53.24$ \\
\hline 15 & 4.4 & Chandra & $<14.2$ & $<30.5$ & $<199.5$ \\
\hline 16 & 4.4 & Chandra & $<14.3$ & $<25.3$ & $<67.6$ \\
\hline 17 & 4.1 & Chandra & $<20.6$ & $<42.5$ & $<47.9$ \\
\hline 18 & 4.5 & Chandra & $<19.0$ & $<33.6$ & $<107.2$ \\
\hline 19 & 4.6 & Chandra & $10.3 \pm 4.9$ & $15.0 \pm 8.7$ & $11.7 \pm 5.6$ \\
\hline 20 & 4.7 & Chandra & $<23.9$ & $<42.3$ & $<173.8$ \\
\hline 21 & 4.5 & Chandra & $23.4 \pm 7.3$ & $30.1 \pm 6.4$ & $25.1 \pm 7.8$ \\
\hline 22 & 7.0 & $X M M-N$ & $12.7 \pm 4.7$ & $13.4 \pm 5.2$ & $6.5 \pm 2.4$ \\
\hline 23 & 4.4 & Chandra & $<14.2$ & $<29.5$ & $<47.59$ \\
\hline 24 & 4.8 & Chandra & $<17.9$ & $<37.8$ & $<34.7$ \\
\hline 25 & 4.6 & Chandra & $15.3 \pm 5.2$ & $22.1 \pm 5.5$ & $44.7 \pm 15.2$ \\
\hline 26 & 4.4 & Chandra & $<23.6$ & $<49.8$ & $<182.0$ \\
\hline 27 & 68.4 & Chandra & $<2.1$ & $<3.7$ & $<2.3$ \\
\hline 28 & 4.4 & Chandra & $<13.7$ & $<23.6$ & $<24.5$ \\
\hline 29 & 4.7 & Chandra & $<22.0$ & $<25.2$ & $<53.7$ \\
\hline 30 & 4.5 & Chandra & $<20.0$ & $<45.3$ & $<107.2$ \\
\hline 31 & 66.9 & Chandra & $1.4 \pm 0.5$ & $2.0 \pm 0.8$ & $1.5 \pm 0.6$ \\
\hline 32 & 67.8 & Chandra & $2.3 \pm 0.6$ & $3.6 \pm 0.8$ & $2.2 \pm 0.6$ \\
\hline 33 & 66.6 & Chandra & $2.6 \pm 0.7$ & $4.4 \pm 0.8$ & $3.0 \pm 0.8$ \\
\hline 34 & 4.7 & Chandra & $6.3 \pm 3.7$ & $10.4 \pm 6.1$ & $<8.5$ \\
\hline 35 & 4.6 & Chandra & $<14.4$ & $<25.5$ & $<19.5$ \\
\hline 36 & 14.2 & $X M M-N$ & $9.1 \pm 4.1$ & $5.2 \pm 1.3$ & $5.2 \pm 2.4$ \\
\hline 37 & 4.7 & Chandra & $<14.2$ & $<31.9$ & $<6.3$ \\
\hline 38 & 17.6 & $X M M-N$ & $<3.0$ & $<4.3$ & $<4.8$ \\
\hline 39 & 21.0 & $X M M-N$ & $37.0 \pm 4.8$ & $32.5 \pm 9.7$ & $41.7 \pm 5.4$ \\
\hline 40 & 21.1 & $X M M-N$ & $30.6 \pm 4.3$ & $20.0 \pm 2.1$ & $14.1 \pm 2.0$ \\
\hline 41 & 26.8 & $X M M-N$ & $21.9 \pm 3.4$ & $25.1 \pm 5.4$ & $45.7 \pm 7.1$ \\
\hline 42 & 14.5 & $X M M-N$ & $<2.0$ & $<2.7$ & $<2.5$ \\
\hline 43 & 14.1 & $X M M-N$ & $46.3 \pm 6.8$ & $20.4 \pm 3.0$ & $38.0 \pm 5.6$ \\
\hline 44 & 22.0 & $X M M-N$ & $49.7 \pm 5.4$ & $26.5 \pm 4.2$ & $19.1 \pm 2.1$ \\
\hline
\end{tabular}

Column: (1) ID; (2) total effective exposure in ks; (3) telescope (XMM-Newton or Chandra); (4) background-subtracted count rate in the T band in units of $10^{-4}$ Count/s; (5) flux in the $\mathrm{T}$ band in units of $10^{-15} \mathrm{erg} \mathrm{s}^{-1} \mathrm{~cm}^{-2} ;(6)$ logarithm of the $F_{2-10} / F_{\mathrm{R}}$ ratio.

the T band span from $1.8 \times 10^{-15}$ to $1.1 \times 10^{-13} \mathrm{erg} \mathrm{s}^{-1} \mathrm{~cm}^{-2}$ with a distribution mean value of $\sim 2 \times 10^{-14} \mathrm{erg} \mathrm{cm}^{-2} \mathrm{~s}^{-1}$.

\section{X-ray spectral properties}

In order to characterize their spectral properties (with particular emphasis on the absorbing column density $N_{\mathrm{H}}$ ), we selected the sources showing a detection in at least one of the following energy bands: soft (i.e. $0.5-2 \mathrm{keV}, \mathrm{S}$ band hereafter) or hard (i.e. $2-10 \mathrm{keV}, \mathrm{H}$ band). This criterion indeed allows to derive the hardness ratio (HR) and, therefore, provide an estimate of the absorption column density even if just a handful of counts are collected during an observation. This condition was satisfied by 23 out of 44 sources, i.e. $\sim 50 \%$ of the sample and, for the 12 brightest sources, i.e. with $\geq 40$ net counts in the $\mathrm{T}$ band, we were also able to perform a basic spectral analysis (we refer hereafter to this sample of 23 EDOGs as the "X-ray sample").

\subsection{Spectral analysis}

All sources detected with a number of $\mathrm{T}$ band counts $\geq 40$ are observed with XMM-Newton. Spectra were acquired from the 
Table 3. X-ray spectral properties of the 23 sources showing a detection in at least one of the two energy bands, $\mathrm{S}$ and $\mathrm{H}$.

\begin{tabular}{|c|c|c|c|c|c|c|c|c|c|}
\hline $\begin{array}{l}\text { ID } \\
\text { (1) }\end{array}$ & $\begin{array}{c}\mathrm{CR}_{0.5-2} \\
(2)\end{array}$ & $\begin{array}{l}\mathrm{CR}_{2-10} \\
\text { (3) }\end{array}$ & $\begin{array}{l}\mathrm{HR} \\
(4)\end{array}$ & $\begin{array}{c}\text { Flux }_{2-10} \\
(5)\end{array}$ & $\begin{array}{l}N_{\mathrm{H}} \\
(6)\end{array}$ & $\begin{array}{c}\log \left(L_{\mathrm{X}, \mathrm{obs}}\right) \\
(7)\end{array}$ & $\begin{array}{c}\log \left(L_{X}\right) \\
(8)\end{array}$ & $\begin{array}{c}\log \left(\lambda L_{5.8} / L_{2-10}\right) \\
(9)\end{array}$ & $\begin{array}{c}\text { Analysis } \\
(10)\end{array}$ \\
\hline 1 & $19.4 \pm 3.4$ & $6.1 \pm 2.5$ & $-0.52 \pm 0.19$ & $10.2_{-40}^{+3.8}$ & $1.1_{-07}^{+1.4}$ & 43.68 & 43.72 & 1.41 & SP \\
\hline 4 & $1.7 \pm 0.8$ & $2.2 \pm 0.9$ & $0.13 \pm 0.31$ & $8.5_{-4.2}^{+5.9}$ & $19.9_{-14.4}^{+37.8}$ & 43.90 & 44.31 & 1.31 & SP \\
\hline 5 & $2.7 \pm 1.3$ & $1.8 \pm 1.2$ & $-0.25 \pm 0.53$ & $2.2_{-0.6}^{+2.2}$ & $<14.3$ & 42.93 & 43.03 & 2.1 & SP \\
\hline 6 & $21.1 \pm 2.5$ & $63.7 \pm 4.3$ & $0.49 \pm 0.07$ & $113.1_{-30.0}^{+17.0}$ & $10.8_{-38}^{+3.6}$ & 44.49 & 44.71 & 0.95 & SP \\
\hline 7 & $4.0 \pm 2.8$ & $7.4 \pm 3.9$ & $0.30 \pm 0.44$ & $14.2_{-3.0}^{+3.0}$ & $25.0_{-23.0}^{+35.8}$ & 44.40 & 44.48 & 1.43 & HR \\
\hline 8 & $<6.9$ & $4.8 \pm 4.1$ & $>-0.53$ & $3.4_{-1.6}^{+1.6}$ & $>0.1$ & 43.13 & $>43.13^{a}$ & 1.29 & HR \\
\hline 9 & $1.8 \pm 1.6$ & $2.8 \pm 1.9$ & $0.21 \pm 0.54$ & $\begin{array}{l}5.3_{-1.9}^{+1.9} \\
\end{array}$ & $25.0_{-240}^{+60.0}$ & 44.08 & 44.15 & 1.47 & HR \\
\hline 10 & $1.0 \pm 0.9$ & $<1.5$ & $<0.25$ & $<2.3_{-1.4}^{+1.4}$ & $<6.0$ & 42.82 & 42.87 & 1.76 & HR \\
\hline 11 & $5.0 \pm 2.3$ & $3.5 \pm 2.4$ & $-0.18 \pm 0.39$ & $5.8_{-2.7}^{+2.6}$ & $4.9_{-4.0}^{+9.9}$ & 43.40 & 43.57 & 1.73 & SP \\
\hline 12 & $1.9 \pm 1.6$ & $5.7 \pm 2.5$ & $0.50 \pm 0.44$ & $27.1_{-3.9}^{-4.18}$ & $267.9_{-144.8}^{+27.0}$ & 43.40 & 45.16 & 0.34 & SP \\
\hline 19 & $2.2 \pm 1.3$ & $8.6 \pm 4.4$ & $0.60 \pm 0.62$ & $12.6_{-7.9}^{+7.9}$ & $\begin{array}{l}25.0_{-17.0}^{+75.0} \\
x^{2}\end{array}$ & 44.10 & 44.92 & 1.76 & HR \\
\hline 21 & $13.0 \pm 5.3$ & $10.6 \pm 4.9$ & $-0.10 \pm 0.31$ & $24.8_{-64}^{+6.9}$ & $4.0_{-35}^{+8.0}$ & 44.19 & 44.21 & 1.05 & HR \\
\hline 22 & $5.9 \pm 3.0$ & $6.7 \pm 3.7$ & $0.06 \pm 0.37$ & $11.3_{-4.2}^{-6.4}$ & $13.0_{-11.0}^{+3.5}$ & 44.35 & 44.39 & 1.56 & HR \\
\hline 25 & $10.9 \pm 3.7$ & $4.5 \pm 5.0$ & $-0.42 \pm 0.43$ & $18.6_{-3.8}^{+3.8}$ & $<45.0$ & 45.26 & 45.30 & 1.33 & HR \\
\hline 31 & $<2.2$ & $0.9 \pm 0.4$ & $>-0.15$ & $1.7_{-0.7}^{+0.7^{\circ}}$ & $>0.1$ & 43.24 & $>43.24^{a}$ & 1.70 & HR \\
\hline 32 & $0.7 \pm 0.3$ & $1.8 \pm 0.5$ & $0.49 \pm 0.31$ & $3.1_{-0.5}^{+0.5}$ & $75.0_{-40.0}^{+75.0}$ & 44.10 & 44.53 & 2.23 & HR \\
\hline 33 & $0.7 \pm 0.3$ & $1.9 \pm 0.6$ & $0.48 \pm 0.28$ & $3.8_{-0.6}^{+0.5}$ & $60.0_{-30.0}^{+45.0}$ & 44.10 & 44.59 & 1.82 & HR \\
\hline 36 & $3.7 \pm 2.5$ & $9.4 \pm 3.8$ & $0.43 \pm 0.40$ & $4.3_{-1.2}^{+6.6}$ & $<28.0$ & 43.01 & 43.06 & 1.21 & SP \\
\hline 39 & $11.2 \pm 2.8$ & $25.9 \pm 3.9$ & $0.40 \pm 0.14$ & $29.9_{-13.4}^{-7.2}$ & $6.7_{-2.4}^{+3.5}$ & 43.65 & 43.85 & 1.03 & SP \\
\hline 40 & $23.2 \pm 3.5$ & $6.9 \pm 2.3$ & $-0.53 \pm 0.16$ & $9.3_{-13}^{+2.1 .4}$ & $<1.5$ & 43.88 & 43.90 & 1.34 & SP \\
\hline 41 & $4.6 \pm 1.9$ & $17.5 \pm 2.8$ & $0.58 \pm 0.18$ & $23.7_{-8.1}^{+6.9}$ & $11.9_{-5.0}^{+8.7}$ & 43.88 & 44.05 & 0.46 & SP \\
\hline 43 & $40.7 \pm 5.8$ & $3.3 \pm 3.0$ & $-0.81 \pm 0.18$ & $12.0_{-27}^{+6.6}$ & $<0.3$ & 44.44 & 44.45 & 1.04 & SP \\
\hline 44 & $31.2 \pm 4.1$ & $18.4 \pm 3.6$ & $-0.26 \pm 0.11$ & $19.5_{-75}^{+2.9}$ & $1.7_{-0.8}^{+1.1}$ & 43.94 & 44.01 & 1.24 & SP \\
\hline
\end{tabular}

Column: (1) ID number; (2) count rate in the S band in units of $10^{-4}$ count/s; (3) count rate in the $\mathrm{H}$ band in units of 10 $0^{-4}$ count/s; (4) Hardness Ratio between the $\mathrm{H}$ band and the $\mathrm{S}$ band computed with the formula $\mathrm{H}-\mathrm{S} / \mathrm{H}+\mathrm{S}$; (5) $\mathrm{H}$ band flux in units of $10^{-15} \mathrm{erg} \mathrm{s}^{-1} \mathrm{~cm}^{-2}$; (6) rest-frame column density $N_{\mathrm{H}}$ in units of $10^{22} \mathrm{~cm}^{-2}$; (7) logarithm of the observed luminosity in the $\mathrm{H}$ band $\left(\mathrm{erg} \mathrm{s}^{-1}\right)$; (8) logarithm of the absorption-corrected luminosity in the $\mathrm{H}$ band ( $\mathrm{erg} \mathrm{s}^{-1}$ ); (9) logarithm of the $\lambda L_{5.8 \mu \mathrm{m}} / L_{2-10}$ ratio; (10) analysis method: SP for spectral fitting, HR for hardness ratio. For Chandra data the $2-10 \mathrm{keV}$ count rate and flux was extrapolated from the $2-8 \mathrm{keV}$ value assuming $\Gamma=1.9$.

${ }^{a}$ For these sources we computed the absorption-corrected luminosity using the lower limit on the $N_{\mathrm{H}}$ value. Accordingly, the intrinsic luminosity shown here is also a lower limit.

cleaned event files of each EPIC camera, i.e. PN, MOS1 and MOS2.

Source counts were extracted from circular regions with a radius in the range of 10-20 arcsec. The radius was chosen in order to optimize the signal-to-noise ratio $(\mathrm{S} / \mathrm{N})$ for each source, but in same cases it was limited by the presence of nearby sources or CCD gaps. Appropriate response and ancillary files for all the EPIC cameras were created using RMFGEN and ARFGEN tasks in the XMM-Newton SAS, respectively. In case of multiple observations of the same source, we combined the source and the background spectra from different exposures using the tool mathpha in order to improve the photon statistics. In doing so we also computed the averages of Response Matrix Files and Ancillary Region Files, weighted for exposure times, using the tools addrmf and addarf. We performed the spectral analysis for 12 sources having net $\mathrm{T}$ band counts in the range 40-600 (with a median value of $\sim 80$ counts). All the fits discussed in this paper include the absorption due to the line-of-sight Galactic column density (Dickey \& Lockman 1990).

For the nine sources with a number of counts $<150$ we used the C-statistic minimization method (Cash 1979), being particularly well-suited for low-count spectra. This is a maximum likelihood method that allows the use of unbinned data. Since it assumes that the counts collected in a given channel follow a Poisson distribution, it cannot be applied to background-subtracted data. Therefore, before performing the spectral modeling we accurately parametrize the background spectrum, extracted from a large source-free region for each source, with a model consisting of a broken power-law plus Gaussian lines, as suggested by Lumb et al. (2002). Then we included this background model in the source spectral fit (fixing the values of the parameters and rescaling the normalizations to the area of the source extraction region). We assumed an absorbed power-law as spectral fitting model. Due to the low number of counts we decided to fix the power-law photon index $\Gamma$ to 1.9 , i.e. the average value found for large samples of quasars in the 2-10 keV band (e.g. Reeves \& Turner 2000; Piconcelli et al. 2005, and references therein)

For the remaining three sources (namely source Nos. 6, 39, 44) with $>150$ net spectral counts we were able to perform the spectral analysis using the $\chi^{2}$ minimization method. In particular, the large number of collected counts for the source No. 6 (SWIRE2 J021749.00-052306.9) allowed to significantly detect an Fe $\mathrm{K} \alpha$ emission line at $\sim 6.4 \mathrm{keV}$ in this high-luminosity $\left(L_{2-10} \approx 5 \times 10^{44} \mathrm{erg} \mathrm{s}^{-1}\right)$ obscured quasar at $z \sim 1$ (see Sect. 6.2 for further details). Table 3 reports X-ray counts and spectral parameters for these 12 sources (labeled with SP). We also report the observed $L_{2-10}$, the absorption corrected $L_{2-10}$ and the ratio

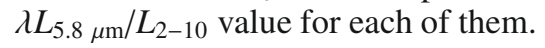

\subsection{Hardness ratio analysis}

For the 11 sources detected with $<40 \mathrm{~T}$ band counts we calculated the background-subtracted count-rate in the $\mathrm{S}$ and $\mathrm{H}$ 

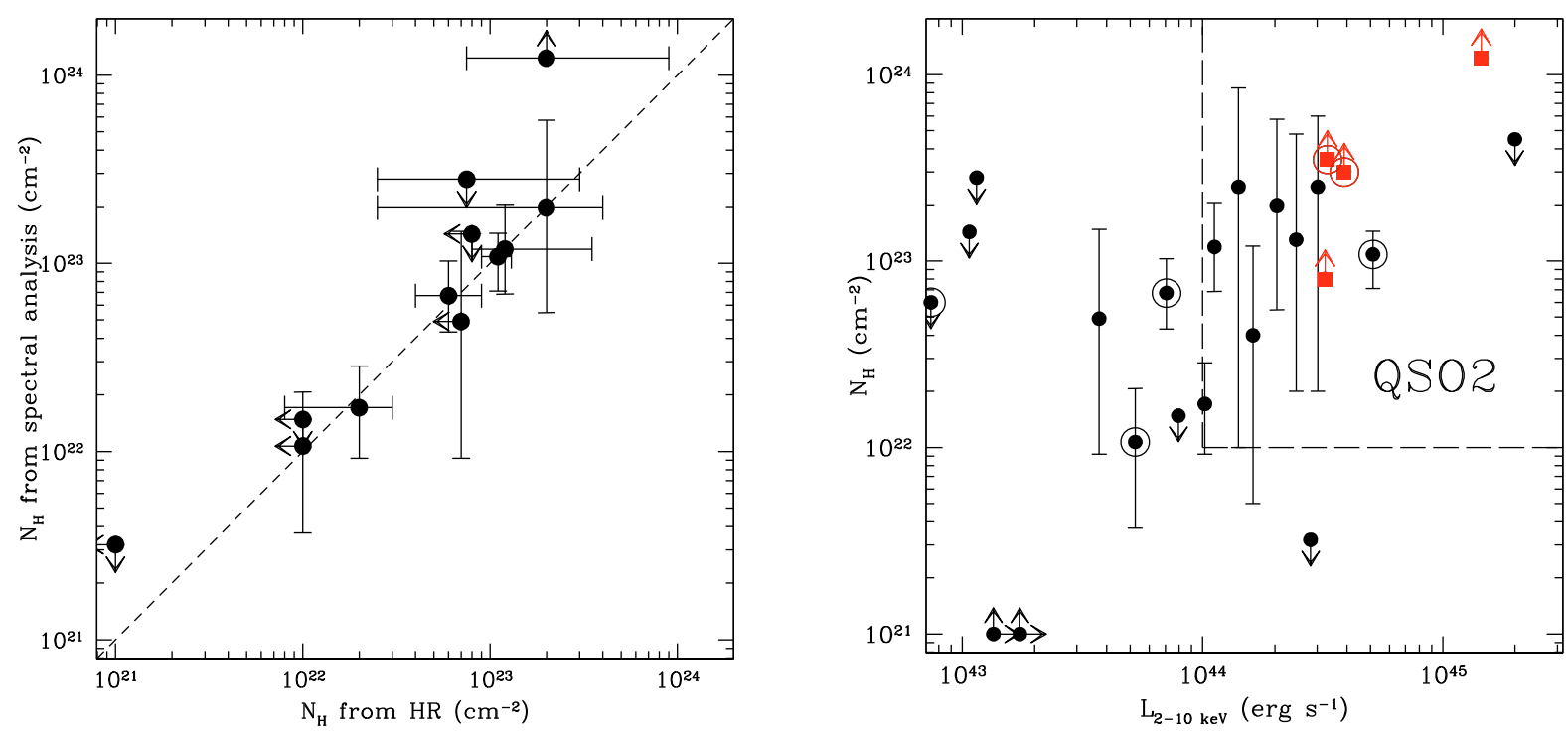

Fig. 4. a) Left panel: comparison of the best fit values of $N_{\mathrm{H}}$ to those obtained with $\mathrm{HR}$ technique, for the 12 sources with available spectral analysis. b) Right panel: distribution of column density $N_{\mathrm{H}}$ as a function of the $L_{2-10}$. Filled squares indicate sources consistent with being Compton Thick AGNs. For these sources we plot the lowest value at $90 \%$ confidence level for the $N_{\mathrm{H}}$ parameter. Sources with a detection only in the Hard band have a lower limit both in the $N_{\mathrm{H}}$ and $L_{2-10}$ values. Circled points represent the spectroscopically-identified sources. Dashed line marks the locus for sources with $N_{\mathrm{H}}$ and $L_{2-10}$ values typical of QSO2s $\left(N_{\mathrm{H}}>10^{22} \mathrm{~cm}^{-2}, L_{2-10}>10^{44} \mathrm{erg} \mathrm{s}^{-1}\right)$.

band. We computed the HR value, defined by the formula $C_{\mathrm{H}^{-}}$ $C_{\mathrm{S}} / C_{\mathrm{H}}+C_{\mathrm{S}}$, where $C_{\mathrm{S}}\left(C_{\mathrm{H}}\right)$ is the net count-rate in $\mathrm{S}(\mathrm{H})$ band. The HR provides an indication about the flatness of an X-ray spectrum and, therefore, it can be used for an estimate of the strength of X-ray obscuration, since the photoelectric absorption mainly affects the soft X-ray portion of the spectrum (i.e. photoelectric absorption cross-section $\sigma(E) \propto E^{-3.5}$ ).

To evaluate the absorbing column density $N_{\mathrm{H}}$, we created a simulated spectrum adopting as model a power law (with photon index $\Gamma=1.9$ ) modified by a rest-frame absorption. We varied the column density $N_{\mathrm{H}}$ in the range $20<\log \left(N_{\mathrm{H}}\right)<24 \mathrm{~cm}^{-2}$ by steps of $\Delta \log \left(N_{\mathrm{H}}\right)=0.2$, and the redshift in the range $0 \leq z \leq 3$ by steps of $\Delta z=0.5$. By computing the HR of the simulated spectrum for each value of $N_{\mathrm{H}}$ and $z$, we created a set of curves corresponding to different values of $N_{\mathrm{H}}$ in the plane $z$ versus HR that allowed us to evaluate the absorption $N_{\mathrm{H}}$ for each source. Thus, we were able to compute both the observed and the absorption corrected $L_{2-10}$. For 2 source (Nos. 8 and 31) with a positive detection only in the $\mathrm{H}$ band, we obtained a lower limit on HR and, hence, $N_{\mathrm{H}}$. The latter was used in the computation of the absorption corrected luminosity Table 3 reports X-ray counts and spectral parameters for these 11 source (labeled HR).

Previous works (e.g. Perola et al. 2004) suggested that the $N_{\mathrm{H}}$ values obtained through the HR technique tend to be overestimated. In order to check the reliability of the HR technique in case of a small number of counts, we compared the $N_{\mathrm{H}}$ inferred by spectral analysis with the $N_{\mathrm{H}}$ value obtained using the method outlined above for the subsample of 12 sources (see Sect. 4.1). The values of $N_{\mathrm{H}}$ determined for each sources are in good agreement within errors as shown in Fig. 4a. Notably, the only source whose $N_{\mathrm{H}}$ estimate does not match the one-toone relationship is the Compton-thick QSO2 candidate SWIRE2 J022003.95-045220.4 (i.e. source No. 12, see Appendix for further details). This suggests that, due to the intrinsically different spectral shape of Compton-thick sources, the HR analysis can lead to underestimate the $N_{\mathrm{H}}$ value in these sources.

\subsection{Absorption properties}

All the sources in the X-ray sample show a hard X-ray luminosity value typical of AGNs, i.e. $L_{2-10} \gg 10^{42} \mathrm{erg} \mathrm{s}^{-1}$. We found that the vast majority (i.e. $~ 95 \%$ ) of the 23 EDOGs with X-ray spectral data are consistent with being obscured by neutral gas with an intrinsic column density of $N_{\mathrm{H}} \geq 10^{22} \mathrm{~cm}^{-2}$ (Fig. 4b). Unfortunately, the combination of large $z$ values and, mostly, the limited photon statistics do not allow us to test the presence of warmly ionized absorbing gas in our sources. In particular the fraction of X-ray obscured EDOGs showing a cold absorption column density strictly larger than $10^{22} \mathrm{~cm}^{-2}$ is $\sim 55 \%$. The uncertainties on this fraction is due to the large errors on the $N_{\mathrm{H}}$ value for the sources detected with a small number of counts. Table 4 lists the source breakdown according to the level of absorption. This table, as well as Fig. 4b, also reveals the common presence ( $\sim 55 \%)$ of QSO2s among the EDOGs in the $\mathrm{X}$-ray sample. This finding highlights the efficiency of our MIR selection criteria in collecting a large number of these elusive AGNs, which are easily missed in optical and X-ray surveys. This makes promising their application to large samples of X-ray well-exposed EDOGs with the goal of studying the properties of the absorption at the brightest end of the AGN X-ray luminosity distribution. It is also worth noting that the sources with the highest absorption column densities fall within the QSO2 locus in the $N_{\mathrm{H}}$ versus $2-10 \mathrm{keV}$ luminosity plane.

To further test the real capability of our MIR selection criteria in gathering QSO2s, we compared these results with those obtained for a control sample consisting of 20 randomly-selected sources from the same SWIRE fields taken into account in our work. We picked out these sources for having a hard X-ray flux range $\left(F_{2-10} \approx 10^{-15}-10^{-13} \mathrm{erg} \mathrm{cm}^{-2} \mathrm{~s}^{-1}\right)$ and a redshift distribution similar to those of our X-ray sample, regardless of their MIR and optical properties. Photometric redshifts for these sources are taken from Rowan-Robinson et al. (2008) (13 sources) or have been derived from optical and IR photometric data (7 sources). Spectral parameters were derived by the 

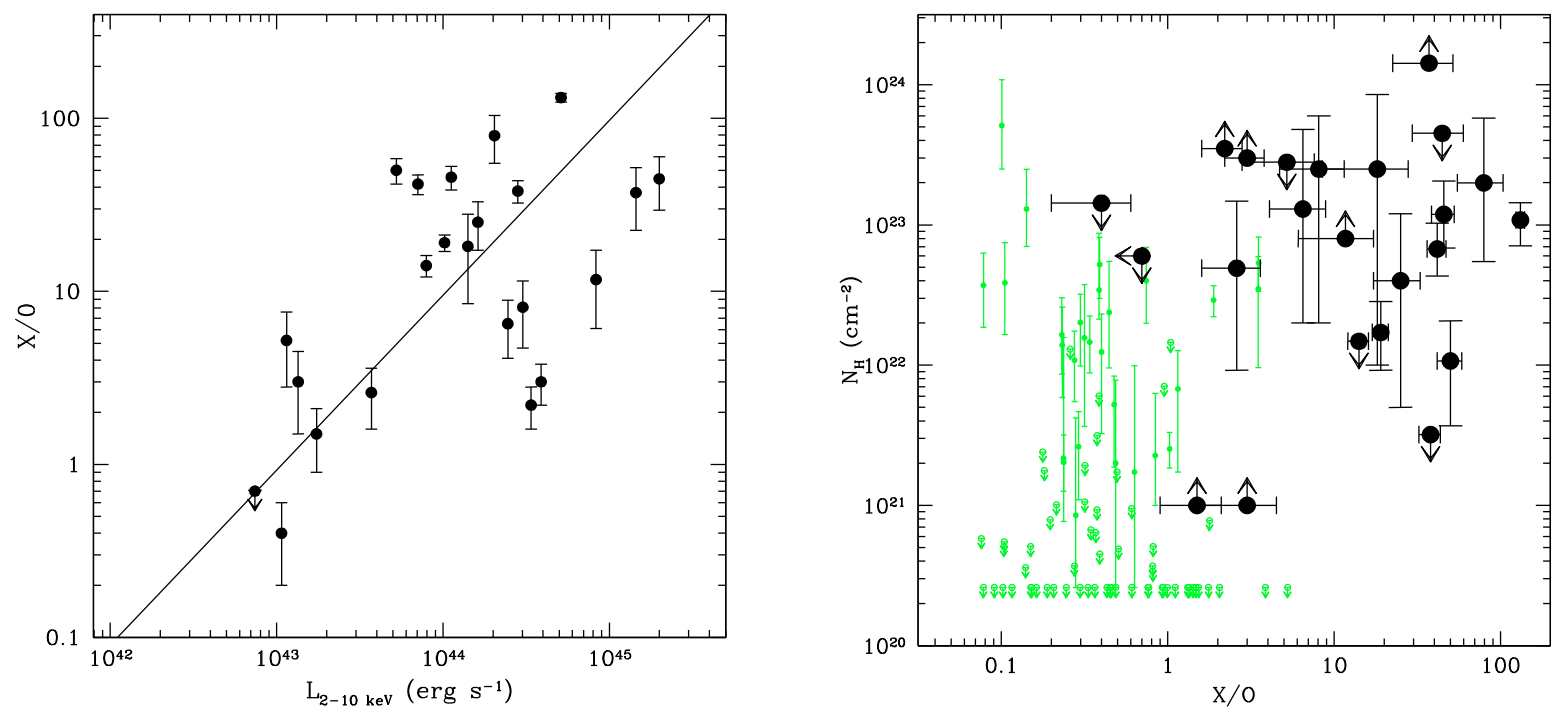

Fig. 5. a) Left panel: $F_{2-10} / F_{\mathrm{R}}$ ratio as a function of the $2-10 \mathrm{keV}$ luminosity for the X-ray sample. The solid line is the best $\log \left(F_{2-10} / F_{\mathrm{R}}\right)-$ $\log \left(L_{2-10 \mathrm{keV}}\right)$ linear regression obtained by Fiore et al. (2003) for a large sample of optically obscured AGNs (see Sect. 4 for details). b) Right panel: $F_{2-10} / F_{\mathrm{R}}$ ratio versus column density $N_{\mathrm{H}}$ for the sources in the X-ray sample (large points). For comparison we also plot the values obtained for a sample of the 99 brightest, hard X-ray selected sources in the wide-area (6 $\left.\mathrm{deg}^{2}\right)$ XMM-LSS survey from Garcet et al. (2007) (small circles).

Table 4. The source breakdown according to the absorption properties. See Sect. 4.3 for details.

\begin{tabular}{|c|c|c|}
\hline Source & X-ray & Control \\
\hline Type & $\begin{array}{c}\text { Sample } \\
\text { (1) }\end{array}$ & $\begin{array}{c}\text { Sample } \\
\text { (2) }\end{array}$ \\
\hline$N_{\mathrm{H}}<10^{22}$ & $13-52 \%$ & $85 \%$ \\
\hline$N_{\mathrm{H}} \geq 10^{22}$ & $48-87 \%$ & $15 \%$ \\
\hline QSO2s & $43-60 \%$ & $5 \%$ \\
\hline N. of Sources & 23 & 20 \\
\hline
\end{tabular}

Column: (1) Percentage of objects for each source type found in our sample (i.e. for the absorbed sources we report the maximum and minimum value derived assuming the best fit value and the lowest value at $90 \%$ confidence level for the $N_{\mathrm{H}}$ parameter, respectively); (2) percentage of objects for each source type in the control sample ( 20 objects).

individual X-ray spectral analysis of each source. The fraction of sources in the control sample with $N_{\mathrm{H}} \geq 10^{22} \mathrm{~cm}^{-2}$ is about $15 \%$. Finally, the fraction of QSO2s is $\sim 5 \%$.

\subsection{X-ray to optical flux ratio}

Optically-selected AGNs typically show an $F_{2-10} / F_{\mathrm{R}}$ flux ratio, in the range $0.1-10$, while $\sim 20 \%$ of AGNs selected in hard X-ray deep surveys has an $F_{2-10} / F_{\mathrm{R}} \geq 10$ (see Alexander et al. 2001; Fiore et al. 2003). Follow-up studies of these EXOs found that most of them are dust-enshrouded AGN at $z \gtrsim 1$ (e.g. Maiolino et al. 2006, and references therein). Fiore et al. (2003) discovered the existence of a striking correlation between $F_{2-10} / F_{\mathrm{R}}$ and $2-10 \mathrm{keV}$ luminosity for non type 1 AGNs whereby the most luminous X-ray sources tend to show the highest $F_{2-10} / F_{\mathrm{R}}$ values. This is due to the fact that Compton-thin AGNs have their nuclear optical/UV light strongly attenuated differently from the X-ray light and, therefore, their $F_{2-10} / F_{\mathrm{R}}$ represents the ratio between the X-ray flux from the AGN and the host galaxy star light, which spans a small luminosity range. Furthermore, the $F_{2-10} / F_{\mathrm{R}}$ value is a proxy for the absorption in AGN (i.e. the higher is $F_{2-10} / F_{\mathrm{R}}$, the higher is $N_{\mathrm{H}}$ ) and, therefore, can be used to select obscured AGNs.
In order to investigate the effects of our MIR/Optical selection on the distribution of $F_{2-10} / F_{\mathrm{R}}$, we computed this ratio for the sources in our sample ${ }^{7}$ (see Table 2). In Fig. 5a we plot the distribution of $F_{2-10} / F_{\mathrm{R}}$ as a function of the $2-10 \mathrm{keV}$ luminosity for the X-ray sample. A clear correlation between $F_{2-10} / F_{\mathrm{R}}$ and hard $\mathrm{X}$-ray luminosity is found, even if characterized by a quite large scatter, in particular for the most X-ray absorbed sources. The solid line in the plot represents the best $\log \left(F_{2-10} / F_{\mathrm{R}}\right)-\log \left(L_{2-10 \mathrm{keV}}\right)$ linear regression obtained by Fiore et al. (2003) from a large sample of X-ray selected, opticallyobscured AGNs.

The $F_{2-10} / F_{\mathrm{R}}$ value as a function of the column density $N_{\mathrm{H}}$ for our X-ray sample is displayed in Fig. 5b. Notably, all but two sources with $F_{2-10} / F_{\mathrm{R}}>10$ show X-ray absorption column densities consistent with being $N_{\mathrm{H}} \gtrsim 10^{22} \mathrm{~cm}^{-2}$. We also report in this diagram the values obtained for a sample of the brightest 99 hard X-ray sources detected in the XMM-LSS survey (e.g. Garcet et al. 2007). This survey covers $\sim 6 \mathrm{deg}^{2}$ and, therefore, is one of the surveys with the widest coverage area in X-rays. This sample of 99 optically identified sources (half of sources show a $z>0.5)$ covers roughly the same range of $2-10 \mathrm{keV}$ flux $\left(4 \times 10^{-15}<F_{2-10}<2 \times 10^{-13} \mathrm{erg} \mathrm{cm}^{-2} \mathrm{~s}^{-1}\right)$ of our X-ray sample, and the $N_{\mathrm{H}}$ values were obtained from individual spectral analysis. From this figure it can be seen that EDOGs have higher values of $F_{2-10} / F_{\mathrm{R}}$ and that also the $N_{\mathrm{H}}$ values are significantly higher, as expected on the basis of Fiore et al. (2003) and Cocchia et al. (2007) results.

\section{Discussion}

\subsection{Efficiency of our MIR selection criteria to collect QSO2s}

Our study was aimed at testing the capability of selection criterion $\left(F_{24 \mu \mathrm{m}} / F_{\mathrm{R}} \geq 2000\right.$ and $\left.F(24 \mu \mathrm{m}) \geq 1.3 \mathrm{mJy}\right)$ to isolate luminous, $X$-ray absorbed quasars at $z \gtrsim 1$. The discovery of a QSO2 in $\sim 55 \%$ of EDOGs in the X-ray sample is the

\footnotetext{
7 For the sources which are undetected in the $2-10 \mathrm{keV}$ band we derived an upper limit in the $F_{2-10} / F_{\mathrm{R}}$ value extrapolating the $\mathrm{H}$ band flux using as underlying spectral model an absorbed $\left(N_{\mathrm{H}}=10^{23} \mathrm{~cm}^{-2}\right)$ power law with $\Gamma=1.9$, e.g. Sect. 3.2 .
} 

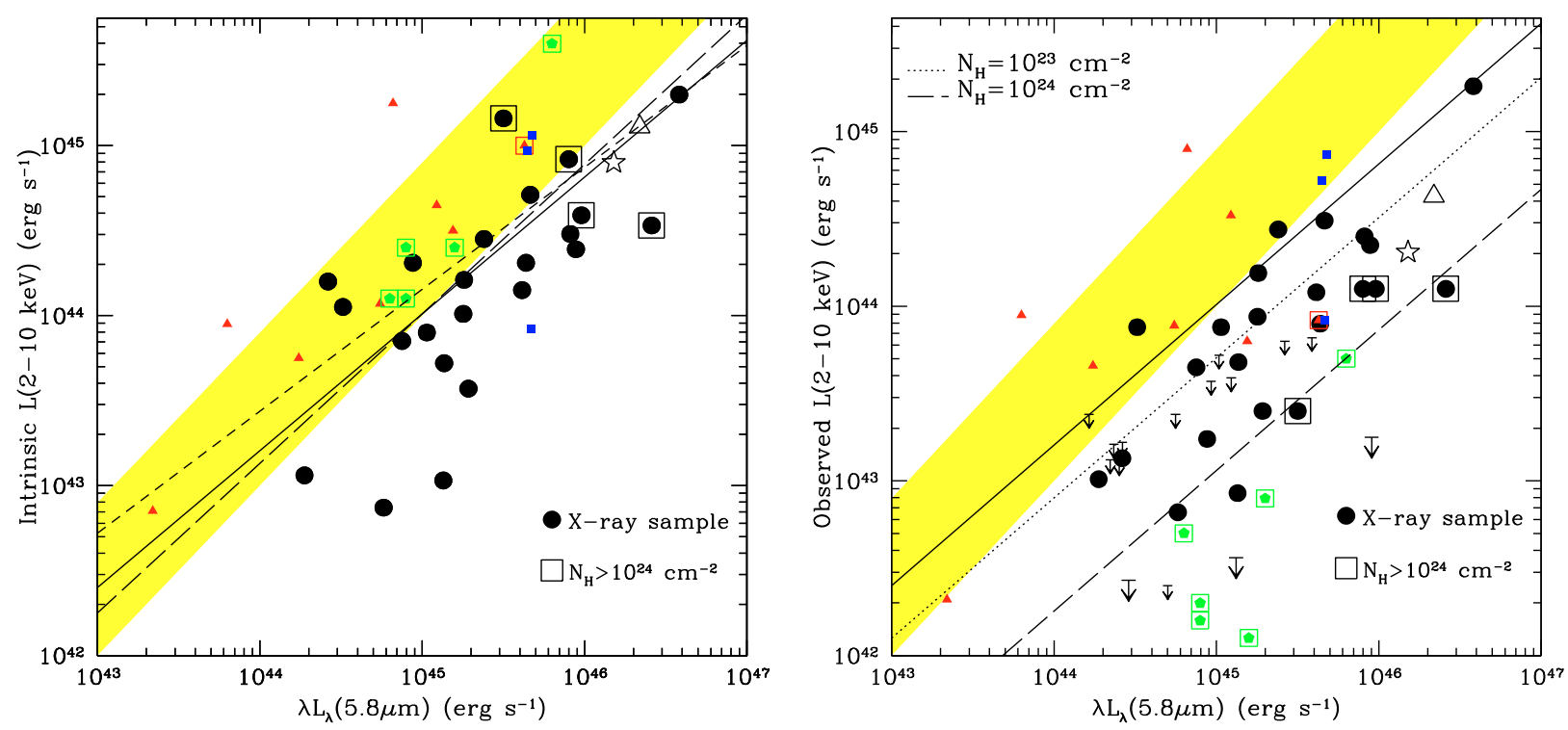

Fig. 6. a) Left panel: $\lambda L_{5.8 \mu \mathrm{m}}$ versus $L_{2-10}$ for sources in our X-ray sample (filled circles), from Polletta et al. (2008a, filled squares), Alexander et al. (2008, filled stars), Sturm et al. (2006, filled triangles). Empty star represents the QSO2 IRAS 09104+4109 (Piconcelli et al. 2007a) and the empty triangle represents the QSO2 4C +24.36 (Johnson et al. 2007). See Sect. 6.2 for further details. Empty squares indicate sources absorbed by a column density of $\log \left(N_{\mathrm{H}}\right) \geq 24 \mathrm{~cm}^{-2}$. Solid line shows the linear regression obtained for sources with $L_{2-10} \geq 10^{43} \mathrm{erg} \mathrm{s}^{-1}$ in our $\mathrm{X}$-ray sample. Short dashed line is the linear regression obtained from COSMOS Compton-thin AGNs (Fiore et al. 2008b). The long dashed line is the combination of the relationships $\lambda L(5100 \AA) / \lambda L(6.7 \mu \mathrm{m})$ and $L_{2-10} / \lambda L(5100 \AA)$ from Maiolino et al. (2007). b) Right panel: $\lambda L_{5.8} \mu \mathrm{m}$ versus the observed $L_{2-10}$. Downward-pointing arrows indicate the $3 \sigma$ upper limit on $L_{2-10}^{\mathrm{Obs}}$ for the X-ray undetected sources (large symbols indicate sources with exposures $\geq 15 \mathrm{ks}$, see Sect. 5.2). Other symbols are as in the previous panel. The solid line represents the linear regression obtained from our X-ray sample, while the dotted and dashed lines represent the ratio $L_{2-10} / \lambda L_{5.8 \mu \mathrm{m}}$ expected for an absorption of $N_{\mathrm{H}}=10^{23}$ and $10^{24} \mathrm{~cm}^{-2}$, respectively. In both panels the shaded area indicates the range of $L_{2-10}-\lambda L_{5.8 \mu \mathrm{m}}$ found for AGNs in the local Universe (Lutz et al. 2004).

most interesting result of our work (e.g. Table 4). It is worth stressing that all the EDOGs with column densities consistent with being Compton-thick fall in the QSO regime, i.e. with a $L_{2-10}>10^{44} \mathrm{erg} \mathrm{s}^{-1}$.

We have thus tested the efficiency of our MIR technique to recover a large number of these elusive objects missed in traditional surveys. They remained poorly-sampled even by blindsearches performed in the deepest X-ray surveys (e.g. Fabian 2002; Tozzi et al. 2006). Our choice of sampling at $F(24 \mu \mathrm{m}) \gtrsim$ $1 \mathrm{mJy}$ appears crucial to identify very luminous AGN-powered objects (as confirmed by the $L_{2-10}>10^{43} \mathrm{erg} \mathrm{s}^{-1}$ measured for the sources in the X-ray sample) and minimize any strong contamination by non-AGN emission (e.g. Weedman et al. 2006; Dey et al. 2008; Sacchi et al., in prep.).

Our findings also highlights the excellent opportunity represented by this simple approach in enabling many important studies concerning the nature of this dust-enshrouded, bright AGN population at high redshifts. In particular, the systematic application of this method to wide-field MIR surveys with appropriately deep and homogeneous X-ray coverage will provide useful insights on largely unexplored issues such as: (i) the demography and surface density of highly obscured quasars; (ii) the multiwavelength SED of distant QSO2s; (iii) the $N_{\mathrm{H}}$ distribution at the largest X-ray luminosities; (iv) the luminosity function of Compton-thin and Compton-thick quasars at $z>1-2$, i.e. near the peak of the AGN activity. Our work may be, therefore, a pathfinder study for the next-generation observatories, when large areas of the sky will be surveyed at faint hard X-ray fluxes.

Our study represents the first attempt to characterize in details the X-ray spectral properties of EDOGs. As expected on the basis of previous works based on analogous (although less severe) MIR selection criteria (Fiore et al. 2008a,b; Georgantopoulos et al. 2008; Polletta et al. 2008a) these extremely red sources are found to harbor X-ray type 2 AGNs. However, this MIR bright galaxy population deserves further and deep investigations in X-rays since it constitutes by far one of the most intriguing recently-discovered class of extragalactic objects. Extraordinary bolometric luminosities, high dust content and redshift distribution of these sources lead to believe that the $E D O G$ phenomenon can be potentially associated with the early stages of quasars evolution as envisaged by Silk \& Rees (1998).

Interestingly, as stressed by Dey et al. (2008), the space density of the $F(24 \mu \mathrm{m}) \geq 1 \mathrm{mJy}$ and $F_{24} \mu \mathrm{m} / F_{\mathrm{R}} \geq 1000$ DOGs is comparable to that of unobscured quasars derived from the MIR luminosity function at $z=2$ (Brown et al. 2006). The brightest DOGs likely are AGN-powered sources deeply obscured both in the optical and soft X-rays and, therefore, cannot be revealed by traditional optical/soft-X surveys, which miss a sizable fraction of the accretion history of the Universe.

In this paper we focus on the most extreme DOGs (representing $\sim 10 \%$ of the $F(24 \mu \mathrm{m}) \geq 1 \mathrm{mJy}$ and $F_{24 \mu \mathrm{m}} / F_{\mathrm{R}} \geq$ 1000 DOGs) as a first step in the exploration of the X-ray spectral properties of this class of MIR bright, dust enshrouded galaxies. We indeed found that the vast majority of the EDOGs harbor an obscured, intrinsically luminous X-ray AGN. It would be very interesting to extend this study to the whole DOG population, in order to provide an accurate estimate of the AGN space density at high $z$.

\subsection{MIR luminosity versus hard $X$-ray luminosity}

In Fig. 6a we compare the 2-10 keV luminosity with the $5.8 \mu \mathrm{m}$ luminosity for sources at the high-luminosity end of the AGN population. Objects from our X-ray sample are indicated with filled circles, while the shaded area represents the region of 
$L_{2-10}-\lambda L_{5.8} \mu \mathrm{m}$ plain derived for AGNs in the local Universe by Lutz et al. (2004). In the figure are also plotted data taken from other samples of QSO2s available in the literature. In particular, we have included: (i) three QSO2s from the Polletta et al. (2008a) sample of $z>1$ objects with $F_{24}>1 \mathrm{mJy}, F_{24 \mu \mathrm{m}} / F_{\mathrm{R}}>$ $400, \lambda L_{5.8 \mu \mathrm{m}}>10^{12} L_{\odot}$ and $10^{21} \lesssim N_{\mathrm{H}} \lesssim 10^{23} \mathrm{~cm}^{-2}$ (namely LH_02, LH_A5 and LH_A6; filled squares); (ii) six Comptonthick $z \sim 2$ QSO2 candidates from Alexander et al. (2008) which are extremely weak (or even undetected) in deep X-ray exposures but show evidence for strong AGN activity in the optical and/or MIR band (filled pentagons); and (iii) eight X-ray selected QSO2s $\left(L_{0.5-10}>10^{44} \mathrm{erg} \mathrm{s}^{-1}\right.$ and $\left.N_{\mathrm{H}}>10^{22} \mathrm{~cm}^{-2}\right)$ from the Sturm et al. (2006) sample (filled triangles).

We have also included in the plot two well-known, very powerful $\left(L_{\mathrm{IR}}>10^{12} L_{\odot}\right)$ dust-enshrouded QSO2s, i.e. IRAS 09104+4109 (empty star, $z=0.442$; e.g. Hines \& Wills 1993; Piconcelli et al. 2007a) and 4C $23.56^{8}$ (empty triangle, $z=2.48$; e.g. Johnson et al. 2006; Seymour et al. 2008). Sources absorbed by a column density of $\log \left(N_{\mathrm{H}}\right) \geq 24 \mathrm{~cm}^{-2}$ are marked with empty squares.

The solid line in this plot represents the linear regression derived for our sample:

$\log \left(L_{2-10}\right)=0.805 \times \log \left(\lambda L_{5.8 \mu \mathrm{m}}\right)+7.785$

once 3 sources with $L_{2-10}<10^{43} \mathrm{erg} \mathrm{s}^{-1}$ were excluded, since their weakness in the hard X-ray band (see Table No. 3) suggests that their $\lambda L_{5.8 \mu \mathrm{m}}$ is starburst-dominated. The long dashed line represents the result (i.e. $\log \left(L_{2-10}\right)=0.88 \times \log \left(\lambda L_{6.7 \mu \mathrm{m}}\right)+$ 4.42) obtained combining the relation between the rest-frame continuum luminosity $\lambda L_{5100 \AA}$ and the MIR luminosity $\lambda L_{6.7} \mu \mathrm{m}$ with the relation between $L_{2-10}$ and $\lambda L_{5100} \AA$, both taken from Maiolino et al. (2007) for a sample of 25 high luminosity (i.e. $\lambda L_{5100 \AA}>10^{46} \mathrm{erg} \mathrm{s}^{-1}$ ) quasars at $2<z<3.5$. The $L_{2-10}-$ $\lambda L_{5.8 \mu \mathrm{m}}$ relationship derived by Fiore et al. (2008b) for a large sample of Compton-thin AGNs in C-COSMOS and CDFS surveys (i.e. $\left.\log \left(L_{2-10}\right)=0.72 \times \log \left(\lambda L_{5.8} \mu \mathrm{m}\right)+11.75\right)$ is marked with the short dashed line.

As expected, we found a correlation between the two luminosities. In particular this relationship is not linear, and the $\lambda L_{5.8 \mu \mathrm{m}} / L_{2-10}$ ratio increases with increasing $\lambda L_{5.8 \mu \mathrm{m}}$. The shift at the highest MIR luminosities from the local Universe relationship suggests a possible evolution of the $\lambda L_{5.8} \mu \mathrm{m} / L_{2-10}$ with redshift. Nonetheless it is evident from this plot that there is a significant scatter in this relationship. This probably means that we have put together a mix of different classes of sources, characterized by different properties and dominant energy mechanisms. The line resulting from Eq. (2) divides the $\lambda L_{5.8 \mu \mathrm{m}}-L_{2-10}$ plane in two regions. The region above this line includes all the X-ray selected QSO2s from Sturm et al. (2006) and the locus typical of AGNs in the local Universe, which was derived by Lutz et al. (2004) from a sample of optically/X-ray bright Seyfert 2 galaxies. Selection effects therefore favor this region to be inhabited by the X-ray/optically "loud" objects. All the sources from the Alexander et al. (2008) fall in this part of the diagram reflecting the fact that they are optically bright enough to be robustly identified via optical spectroscopy even if they likely are Comptonthick QSO2s. The intrinsic X-ray luminosities of these sources

\footnotetext{
8 We re-analyzed the $X M M$-Newton data of $4 \mathrm{C} 23.56$. Our best-fit (an absorbed power law with $N_{\mathrm{H}}=4.4_{-2.4}^{+3.7} \times 10^{23} \mathrm{~cm}^{-2}$ and $\Gamma$ fixed to 1.9 plus a soft $(\Gamma \equiv 3)$ unabsorbed power law) is consistent with those reported by Johnson et al. (2006), confirming the QSO2 nature of this high- $z$ radiogalaxy. We derived a $2-10 \mathrm{keV}$ intrinsic(observed) luminosity of $L_{2-10}=1.3(0.4) \times 10^{45} \mathrm{erg} \mathrm{s}^{-1}$.
}

was indeed estimated on the basis of the luminosity of optical emission lines. Two out of three objects taken from the Polletta et al. (2008a) sample showing moderate absorption in X-rays $\left(N_{\mathrm{H}} \lesssim 10^{23} \mathrm{~cm}^{-1}\right)$ are located in this area. Interestingly, Sturm et al. (2006) emphasize the absence of dust emission and absorption features in the MIR spectra of their QSO2s unlike ULIRGs, which are very dusty systems with powerful circumnuclear starbursts. This points out that these highly luminous X-ray quasars were able to sweep away dust envelopes from their nuclear environment.

In the context of the evolutionary sequence described in the previous section, it seems possible that most of the objects falling in this region of the $L_{2-10}-\lambda L_{5.8} \mu \mathrm{m}$ plane have already passed through the initial dust-enshrouded phase of quasar activity/galaxy assembly (e.g. Silk \& Rees 1998) and are going to evolve into classical "blue" QSOs traditionally collected in optical/UV surveys. IRAS $09104+4109$ and 4C +24.36, which are respectively IR-and radio-selected, lies outside the locus of local Universe AGNs.

On the contrary, the majority of the sources located below the solid line in Fig. 6a might be objects with dust-absorbed powerful (ULIRG-like) nuclei in which the star-formation activity could significantly contribute to their MIR emission or characterized by intrinsic extreme MIR/X luminosity ratios (e.g. Polletta et al. 2008b; Piconcelli et al. 2009, in preparation). Accordingly, these sources may be the tip of a larger population representing the link between X-ray-selected QSO2s with featureless MIR spectra (e.g. Sturm et al. 2006) and the submillimeter galaxies (SMGs) (e.g. Alexander et al. 2005; Pope et al. 2008b) which are well-known sites of strong star-formation activity. In particular, these EDOGs could belong to the minority fraction of SMGs with optical-IR emission dominated by a dust-obscured quasar (e.g. Vignali et al. 2009) with MIR colors overlapping those of MIR power-law continuum AGNs (Yun et al. 2008; Pope et al. 2008a). Thus, even if the position of a source in Fig. 6a strongly depends on the criterion of selection adopted, EDOGs may therefore represent a mixed-bag population that approximately come in two flavors, i.e. "X-ray loud" QSO2-like and SMGs-like, which essentially indicate different stages of quasar evolution. However, caution must be used before drawing any firm conclusion from these plots because of the uncertainties on the intrinsic $N_{\mathrm{H}}$ and the AGN luminosity due to the low quality of X-ray data and the use of photometric redshifts for most of the sources considered here.

Figure $6 \mathrm{~b}$ shows the observed $2-10 \mathrm{keV}$ luminosity $\left(L_{2-10}^{\mathrm{Obs}}\right)$ as a function of $\lambda L_{5.8} \mu \mathrm{m}$ for the same sources plotted in Fig. 6 a. The solid line represents the relationship obtained from the X-ray sample (i.e. Eq. (2)) which can be used to estimate the intrinsic hard X-ray luminosity. The dotted and dashed line represent the observed $L_{2-10}^{\mathrm{Obs}}-\lambda L_{5.8} \mu \mathrm{m}$ ratio derived from Eq. (2) in case of a column density of $N_{\mathrm{H}}=10^{23}$ and $N_{\mathrm{H}}=10^{24} \mathrm{~cm}^{-2}$, respectively. Downward-pointing arrows in the plot indicate the $3 \sigma$ upper limit on $L_{2-10}^{\mathrm{Obs}}$ for the X-ray undetected sources in our sample lying in the AGN region of the IRAC color-color diagram (Fig. 1b), i.e. sources showing a power law spectrum and very red MIR colors $\left(\log \left(F_{5.8} / F_{3.6}\right)>0.2\right.$ and $\log \left(F_{8} / F_{4.5}\right)>$ 0.2 ). Hereafter we refer to this group of 15 sources as the Red Sample.

For these $15 \mathrm{X}$-ray undetected AGNs, we converted the upper limit on the $2-10 \mathrm{keV}$ flux (assuming a photon index of $\Gamma=$ 1.9) into the $2-10 \mathrm{keV}$ observed luminosity $L_{2-10}^{\mathrm{Obs}}$. The intrinsic $L_{2-10}$ for these sources was determined from their $\lambda L_{5.8 \mu \mathrm{m}}$, using the relationship in Eq. (2). By the comparison between the observed and the intrinsic values of hard X-ray luminosity 
Table 5. The source breakdown for the X-ray sample, the Red sample, and the total sample. See Sect. 5.2 for details.

\begin{tabular}{lccc}
\hline \hline $\begin{array}{l}\text { Source } \\
\text { Type }\end{array}$ & $\begin{array}{c}\text { X-ray } \\
\text { Sample* } \\
(1)\end{array}$ & Red Sample & $\begin{array}{c}\text { Total } \\
\text { Sample } \\
(2)\end{array}$ \\
\hline$N_{\mathrm{H}}>10^{22}$ & $50 \%$ & $93 \%$ & $66 \%$ \\
CT AGNs & $13 \%$ & $27 \%$ & $18 \%$ \\
QSO2s & $43 \%$ & $27 \%$ & $37 \%$ \\
N. of Sources & 23 & 15 & 38 \\
\hline
\end{tabular}

Percentage of objects for each source type found in the: (Col. 1) X-ray sample (e.g. Sect. 5); (Col. 2) the X-ray undetected Red sample (i.e. sources showing $\log \left(F_{5.8} / F_{3.6}\right)>0.2$ and $\left.\log \left(F_{8} / F_{4.5}\right)>0.2\right)$; (Col. 3) the combined (i.e. X-ray+undetected Red) sample. *For the $\mathrm{X}$-ray sample we considered the conservative values derived using the lowest value in $90 \%$ confidence level for the $N_{\mathrm{H}}$ parameter.

we were able to provide a basic estimate of the column density distribution in the Red Sample. We found that: (i) $93 \%$ of these sources show a $N_{\mathrm{H}} \geq 10^{22} \mathrm{~cm}^{-2}$; (ii) $27 \%$ of them show a column density $N_{\mathrm{H}}>10^{24} \mathrm{~cm}^{-2}$ and are Compton-Thick AGN candidates; and (iii) QSO2s-like objects account for $27 \%$ of the total (see Table 5).

Since $75 \%$ of the Red Sample sources have X-ray effective exposures of $\sim 5 \mathrm{ks}$, the $3 \sigma$ upper limits are weakly constrained. Accordingly, the real values of the column density may likely be significantly higher than the $N_{\mathrm{H}}$ values derived here, which should be considered as lower limits.

A handful of objects in the Red Sample, namely Nos. 2, 3 and 38 , were not detected in X-ray medium/deep (i.e. $>15 \mathrm{ks}$ ) observations (big downward-pointing arrows in Fig. 6b). This fact makes them very interesting objects since they may likely be more obscured than those found in the X-ray sample. In the MIR color-color diagram presented in Fig. 1b, these EDOGs indeed occupy the area with the extreme red colors, i.e. $\log \left(F_{5.8} / F_{3.6}\right)>$ 0.5 and $\log \left(F_{8} / F_{4.5}\right)>0.5$, that Polletta et al. (2008a) consider as the typical one of highly luminous and obscured quasars at $z \gtrsim$ 1. In particular, we derived an $L_{2-10} \geq 10^{44} \mathrm{erg} \mathrm{s}^{-1}$ for sources Nos. 2 and 3 and $L_{2-10} \sim 3 \times 10^{43} \mathrm{erg} \mathrm{s}^{-1}$ for the No. 38, and the estimated $N_{\mathrm{H}}$ value is $\geq 10^{24} \mathrm{~cm}^{-2}$ for all these three sources, making them very likely Compton-Thick QSO2 candidates.

Merging the results for the Red Sample with those previously found for the X-ray sample, we obtained a total sample of 38 sources for which has been possible to infer information on $N_{\mathrm{H}}$ and hard X-ray luminosity (see Table 5). This allows us to improve the statistics and get sounder insights on the X-ray properties $^{9}$ of EDOGs, which basically turn out to host heavily obscured AGNs ( $20 \%$ are consistent to be Compton-thick) with high X-ray luminosity ( $\gtrsim 40 \%$ are QSO2s).

\section{Summary and conclusions}

We exploited the multiwavelength coverage of the large area SWIRE survey to collect low-surface density/high luminosity (i.e. $10^{12} \lesssim L_{\mathrm{Bol}} \lesssim 10^{14} L_{\odot}$ ) objects on the basis of their extreme MIR/Optical flux ratio $\left(F_{24} \mu \mathrm{m} / F_{\mathrm{R}}>2000\right)$ and bright MIR flux density, i.e. $F_{24} \mu \mathrm{m}>1.3 \mathrm{mJy}$ (referred to here as EDOGs). Cross-correlating with publicly available $X M M$ Newton/Chandra observations of 5 SWIRE fields we defined a final sample of 44 EDOGs with X-ray coverage (see Table 1

\footnotetext{
9 For the X-ray sample we considered the percentages derived using the lowest value in $90 \%$ confidence level for the $N_{\mathrm{H}}$ parameter obtained for each source.
}

and Sect. 2). Our study has been focused on the understanding of largely unexplored X-ray spectral properties of this recentlydiscovered class of sources. The main results of this paper can be summarized as follows:

- All but one sources fall within the AGN region of the MIR color-color diagram proposed by Lacy et al. (2004). In particular, the vast majority of EDOGs lies in the obscured luminous QSO locus (Polletta et al. 2008a) characterized by very red colors (Fig. 1b).

- The very bulk of the redshifts, regardless of they are spectroscopic or photometric, are in the range $0.7 \lessgtr z \lesssim 2.5$ (e.g. Sect. 2.3).

- Thanks to the large area sampled $\left(\sim 6 \mathrm{deg}^{2}\right)$, our EDOGs are on average more luminous in the MIR band by a factor of $\geq 0$.5-2 orders of magnitude than their equivalents in the CDFS and COSMOS surveys (see Fig. 3b).

- Twenty-three sources (the so-called X-ray sample, e.g. Sect. 4) have a positive detection either in the soft or in the hard band. The non-detection of the remaining sources in the sample can be mainly attributed to the inadequate X-ray coverage (most of the undetected sources indeed have an exposure $\leq 5 \mathrm{ks}$ ). All the X-ray detected sources have a 2$10 \mathrm{keV}$ luminosity $L_{2-10}>10^{43} \mathrm{erg} \mathrm{s}^{-1}$, whereby they fall well within the AGN X-ray luminosity range (Fig. 4b).

- Our analysis reveals that $50 \%$ of the sources show a column density strictly larger than $10^{22} \mathrm{~cm}^{-2}$. Furthermore $\sim 50 \%$ of them can be classified as QSO2s on the basis of their absorption properties and X-ray luminosity (i.e. $L_{2-10}>10^{44} \mathrm{erg} \mathrm{s}^{-1}$ ).

- At least three EDOGs at $z>1.4$ were found to be very likely Compton-thick QSO2 candidates (e.g. Sect. 5.2). Moreover such deeply buried AGNs may be present in a sizable fraction $(\sim 30 \%$, e.g. Table 5$)$ of the X-ray undetected sources with extreme red colors $\left(\log \left(F_{5.8} / F_{3.6}\right)>0.2\right.$ and $\left.\log \left(F_{8} / F_{4.5}\right)>0.2\right)$, as suggested by their large MIR luminosity (e.g. Sect. 5.2). They therefore deserve further investigation by future properly-planned, deeper X-ray observations.

Our work represents a further step forward in completing the census of SMBHs through cosmic epochs. The application of our selection criteria to a large area survey has allowed to efficiently pick up a large sample of high- $z$ sources, occupying the bright end of the X-ray obscured AGN population. Thus, it is a useful tool to complement the information derived by blind searches for these elusive objects performed in the pencil-beam, deep X-ray surveys (which mostly sample the low-redshift $(z \sim 1)$, Seyfertlike AGN population) making possible the first reliable estimate of AGN "bolometric" luminosity function at high redshifts.

It seems probable that EDOGs might be an early dustembedded phase of the AGN activity, possibly associated with merger events (e.g. Georgantopoulos et al. 2008; Brodwin et al. 2008). Accordingly, an interesting aspect that should be examined in the next future is the FIR/sub-mm emission of these intriguing sources in order to evaluate the contribution of the starformation activity to their large bolometric luminosity, i.e. using an approach similar to that by Pope et al. (2008a) for a sample of DOGs at low $F_{24} \mu \mathrm{m}$ levels $\left(\left\langle F_{24} \mu \mathrm{m}\right\rangle \sim 175 \mu \mathrm{Jy}\right)$.

Acknowledgements. The authors are very grateful to the referee for the exhaustive list of helpful comments and her/his prompt response. We thank for support the Italian Space Agency (contracts ASI-INAF I/023/05/0, ASI I/088/06/0 and PRIN MIUR 2006-02-5203). The authors kindly thank Simonetta Puccetti, Mari Polletta and Roberto Maiolino for many useful comments. Based on observations obtained with XMM-Newton, an ESA science mission with instruments and contributions directly funded by ESA Member States and NASA. 

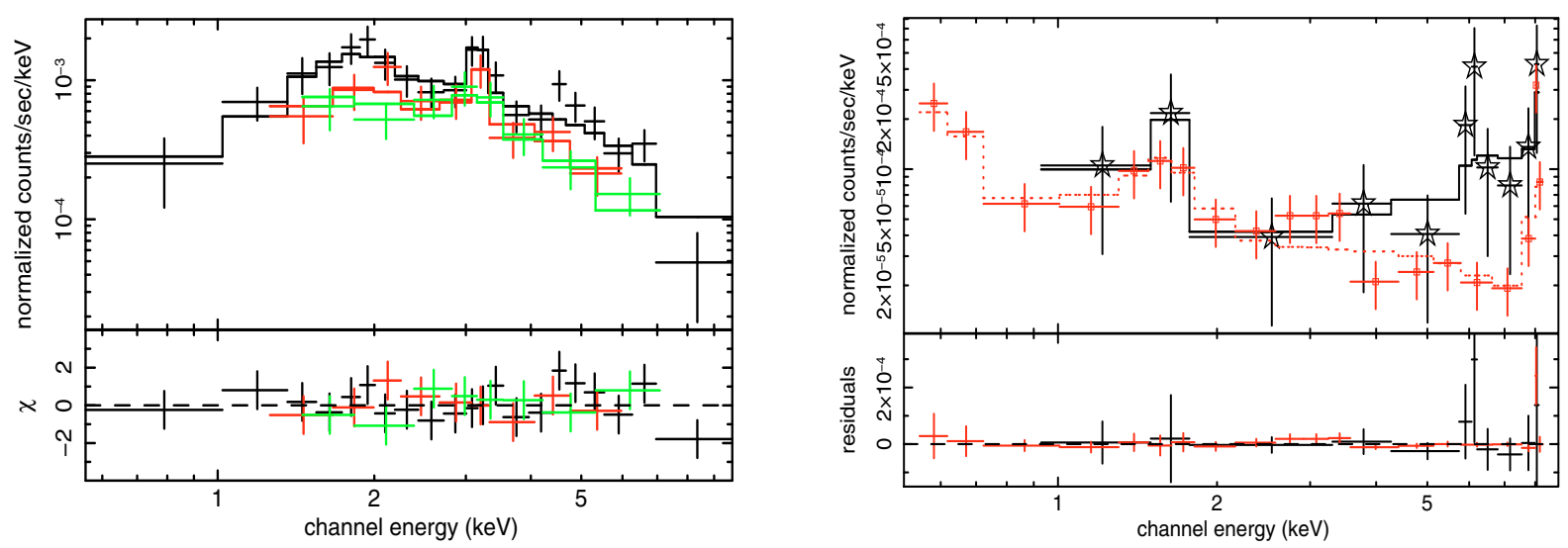

Fig. A.1. a) Left panel: XMM-Newton spectrum of source No. 6 (i.e. SWIRE2 J021749.00-052306.9). The background subtracted spectra of EPIC PN (black), MOS1 (red) and MOS2 (green) are shown. b) Right panel: XMM-Newton spectrum of source No. 12 (i.e. SWIRE2 J022003.95045220.4). The source spectrum (black stars) is superimposed to the background spectrum (red empty squares). The best fit models for the source (black solid line) and the background (red dotted line) are also shown.
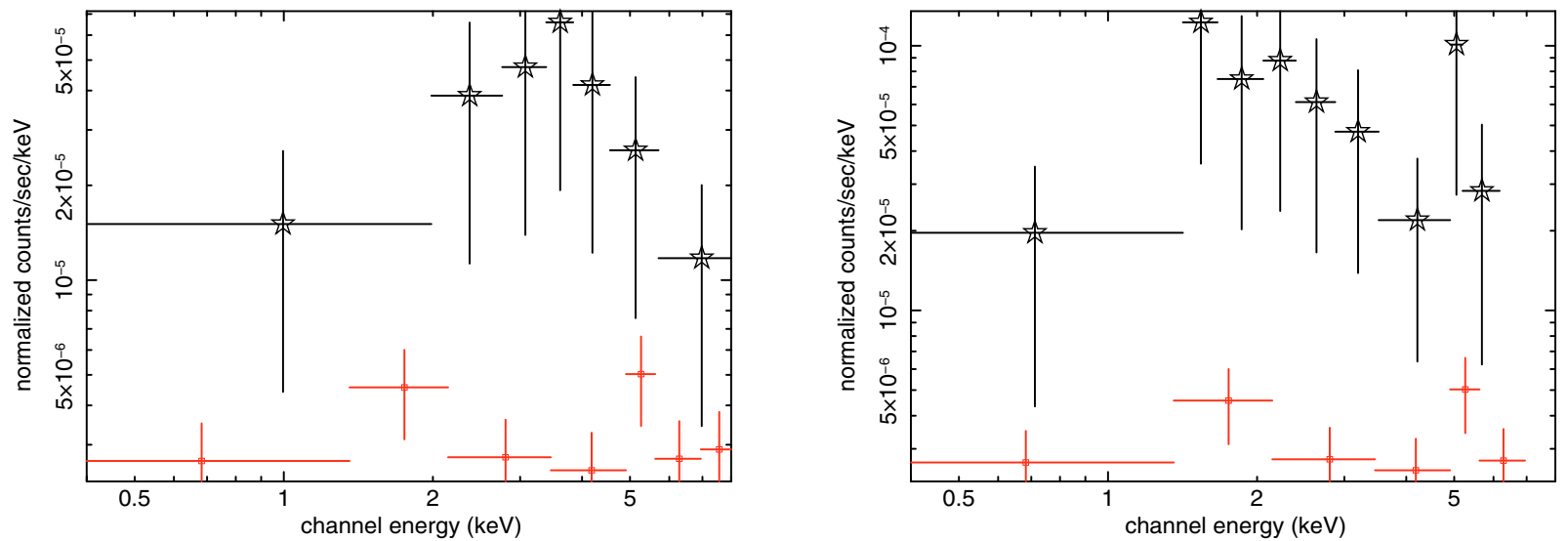

Fig. A.2. a) Left panel: Chandra spectrum of source No. 32 (i.e. SWIRE2 J104409.95+585224.7). b) Right panel: Chandra spectrum of source No. 33 (i.e. SWIRE2 J104528.29+591326.6). Black stars (red empty squares) represent the source (background) binned counts, with 2 (15) counts per bin.

This research has made use of software provided by the Chandra X-ray Center (CXC) in the application package CIAO. This research has made use of the NASA/IPA Extragalactic Database (NED) which is operated by the Jet Propulsion Laboratory, California Institute of Technology, under contract with the National Aeronautics and Space Administration.

\section{Appendix A: Notes on individual sources}

- Source No. 6. This is a narrow-line quasar at $z=0.914$, as shown in on Sect. 2.3.1 on the basis of a Subaru/MOIRCS spectrum. This source is the brightest in our sample, with a 2-10 keV flux of $F_{2-10} \sim 10^{-13} \mathrm{erg} \mathrm{cm}^{-2} \mathrm{~s}^{-1}$. We fitted the EPIC data with a continuum model consisting of a power law modified by an intrinsic neutral absorption component, plus a power law with the same slope and Galactic $N_{\mathrm{H}}$ at soft energies $(\$ 2 \mathrm{keV})$. The latter accounts for the "soft-excess" component, i.e. a mixture of photoionized/scattered emission superimposed upon the primary obscured continuum typically observed in most X-ray obscured AGNs (Turner et al. 1997; Piconcelli et al. 2007b; Cappi et al. 2006). Given the redshift of this $E D O G$, we also included in the best-fit model a Compton reflection component with $R=1$ (where $R$ is the solid angle in units of $2 \pi$ subtended by the reflecting material) as commonly detected in the rest-frame $10-50 \mathrm{keV}$ spectra of AGN (e.g. Risaliti 2002; Reeves et al. 2006). We obtained $\Gamma=1.70 \pm 0.33$ and $N_{H}^{z} \sim 10.8 \times 10^{22} \mathrm{~cm}^{-2}$, implying a 2-10 keV luminosity of $L_{2-10}=5.1 \times 10^{44}$ $\mathrm{erg} \mathrm{s}^{-1}$. The most remarkable result of our analysis is, however, the discovery of an $\mathrm{Fe} \mathrm{K} \alpha$ emission line in the $\mathrm{X}$ ray spectrum of this QSO2 (Fig. A.1a). This line $\left(E_{\mathrm{K} \alpha}=\right.$ $6.29 \pm 0.12 \mathrm{keV})$ is required by the data at $>99.9 \%$ confidence level and shows an equivalent width of $E W=$ $590 \pm 265 \mathrm{eV}$. It represents one of the best examples of $\mathrm{Fe} \mathrm{K} \alpha$ line in a high- $z$ QSO2s detected so far.

- Sources Nos. 12, 32 and 33. These sources (with $z=1.443$, 2.54 and 2.31 respectively) show most of their X-ray counts at $E>2 \mathrm{keV}$ (e.g. Figs. A.1b, A.2a and A.2b) and they are therefore promising deeply buried (possibly Compton-thick) QSO2 candidates. In particular, source No. 12 was detected with $\sim 40(30)$ net counts in the $0.5-10(2-10 \mathrm{keV}) \mathrm{keV}$ band, i.e. at the limit of Cash statistic reliability for the spectral fitting procedure (Sect. 4.1). Fitting these data with a simple spectral model consisting of a power-law with fixed photon index $(\Gamma=1.9)$ modified by an intrinsic absorption component, we obtained a lower limit on the column density of $N_{\mathrm{H}}>1.4 \times 10^{24} \mathrm{~cm}^{-2}$. The corresponding X-ray absorptioncorrected luminosity is $L_{2-10} \sim 1.4 \times 10^{45} \mathrm{erg} \mathrm{s}^{-1}$, making this EDOG one of the best Compton-thick QSO2 candidates in our sample.

Sources Nos. 32 and 33 have only $\sim 20$ net counts, even if they were detected in deep ( $\sim 70 \mathrm{ks})$ Chandra exposures, and almost all of them fall in the hard X-ray band. The HR of 
these sources are therefore very hard ( 0.5, e.g. Table 3$)$ and suggest that they are also highly obscured quasars. We derived column density values of $N_{\mathrm{H}} \gtrsim 3 \times 10^{23} \mathrm{~cm}^{-2}$ for both sources (but keep in mind the large uncertainties on the HR value), confirming thus the results reported in Weedman et al. (2006, sources A4 and A8 in their paper). Beyond providing the spectroscopic redshifts, these authors carried out a detailed multiwavelength study for both sources that allowed to classify them as AGN-powered ULIRGs.

- Source No. 27. This source was undetected in a $\sim 70 \mathrm{ks}$ Chandra exposure, being thus the only EDOG without useful $\mathrm{X}$-ray information in such a deep pointing and, possibly, the weakest X-ray sources in our sample. Its IRAC colors suggest that a sizable part of the IR emission in this source can be powered by star formation (e.g. Sect. 2.2 and Yun et al. 2008). According to Rowan-Robinson et al. (2008), the MIR Spectral Energy Distribution (SED) is indeed dominated by the starburst component. This hypothesis is also supported by the large $F_{24} / F_{8}$ ratio $(\sim 20)$ measured for this $E D O G$. Such a value is similar to those reported for the infrared luminous galaxies at $z \sim 2$ in Yan et al. (2007), for which both deeply embedded starburst and AGN are believed to significantly contribute to the dust heating (e.g. Polletta et al. 2008a).

\section{References}

Alexander, D. M., Brandt, W. N., Hornschemeier, A. E., et al. 2001, AJ, 122, 2156

Alexander, D. M., Smail, I., Bauer, F. E., et al. 2005, Nature, 434, 738

Alexander, D. M., Chary, R. R., Pope, A., et al. 2008, ApJ, in press, [arXiv: 0803.0636]

Alonso-Herrero, A., Perez-González, P. G., Alexander, D. M., et al., 2006, ApJ, 640, 167

Antonucci, R. 1993, ARA\&A, 31, 473

Arnouts, S., Walcher, C. J., Le Fevre, O., et al. 2007, A\&A, 476, 137

Barmby, P., Alonso-Herrero, A., Donley, J. L., et al., 2006, ApJ, 642, 126

Best, P. N., Kauffmann, G., Heckman, T. M, et al., 2005, MNRAS, 362, 25

Brand, K., Dey, A., Weedman, D., et al. 2006, ApJ, 644, 143

Brand, K., Dey, A., Desai, V., et al. 2007, ApJ, 663, 204

Brandt W. N., \& Hasinger, G. 2005, ARA\&A, 43, 827

Brodwin, M., Dey, A., Brown, M. J., et al. 2008, ApJ, 687, L65

Brown, M. J. I., Brand, K., Dey, A., et al. 2006, ApJ, 638, 88

Cappi, M., Panessa, F., Bassani, L., et al. 2006, A\&A, 446, 459

Cardamone, C. N., Urry, C. M., Damen, M., et al. 2008, ApJ, 680, 130

Cash, W. 1979, ApJ, 228, 939

Cocchia, F., Fiore, F., Vignali, C., et al. 2007, A\&A, 466, 31

Comastri, A., 2004, Multiwavelength AGN Surveys, Proceedings of the Guillermo Haro Conference, ed. R. Mujica, \& R. Maiolino (Singapore: World Scientific Publishing Company), 323

Cowie, L. L., Barger, A. J., Bautz, M. W., Brandt, W. N., \& Garmire, G. P. 2003, ApJ, 584, 57

Croton, D. J., Springel, V., White, S. D. M., et al. 2006, MNRAS, 365, 11

Daddi, E., Alexander, D. M., Dickinson, M., et al., 2007, ApJ, 670, 173

Dey, A., Soifer, B. T., Desai, V., et al. 2008, ApJ, 677, 943

Dickey, J. M., \& Lockman, F. J. 1990, ARA\&A, 28, 215

Di Matteo, T., Springel, V., \& Hernquist, L. 2005, Nature, 433, 604

Donley, J. L., Rieke, G. H., Pérez-González, P. G., Rigby, J. R., \& Alonso-Herrero, A. 2007, ApJ, 660, 167

Donley, J. L., Rieke, G. H., Pérez-González, P. G., \& Barro, G. 2008, ApJ, 687, 111

Dwelly, T., \& Page, M. J. 2006, MNRAS, 372, 1755

Elvis, M., Wilkes, B. J., McDowell, J. C., et al. 1994, ApJS, 95, 1

Erlund, M. C., Fabian, A. C., Blundell, K. M., \& Crawford, C. S. 2008, MNRAS, 385, L125

Fabian, A. C. 1999, MNRAS, 308, L39

Fabian, A. C. 2002, ASPC, 258, 185

Feruglio, C., Fiore, F., La Franca, F., et al. 2008, A\&A, 488, 417

Fiore, F., Brusa, M., Cocchia, F., et al. 2003, A\&A, 409, 79

Fiore, F., Grazian, A., Santini, P., et al. 2008a, ApJ, 672, 94

Fiore, F., Puccetti, S., Brusa, M., et al. 2008b, ApJ, in press, [arXiv:0810.0720]

Fontana, A., D’Odorico, S., Poli, S., et al. 2000, AJ, 120, 2206
Fontana, A. 2001, Astrophys. Space Sci. Suppl., 277, 535

Furusawa. H., Kosugi, G., Akiyama, M., et al., 2008, ApJS, 176, 1

Garcet, O., Gandhi, P., Gosset, E., et al. 2007, A\&A, 474, 473

Georgantopoulos, I., Georgakakis, A., \& Akylas, A. 2007, A\&A, 466, 823

Georgantopoulos, I., Georgakakis, A., Rowan-Robinson, M., \& Rovilos, E. 2008, A\&A, 484, 671

Gilli, R., Comastri, A., \& Hasinger, G., 2007, A\&A, 463, 79

Granato, G. L., De Zotti, G., Silva, L., Bressan, A., \& Danese, L. 2004, ApJ, 600,580

Hasinger, G. 2008, A\&A, 490, 905

Hickox, R., Jones, C., Forman, W. R., et al. 2007, ApJ, 671, 1365

Hines, D.C., \& Wills, B. J. 1993, ApJ, 415, 82

Hopkins, P. F., Hernquist, L., Cox, T. J., Di Matteo, T., \& Springel, V. 2006, ApJS, 163, 1

Houck, J. R., Soifer, B. T., Weedman, D., et al. 2005, ApJ, 622, L105

Johnson, O., Almaini, O., Best, P. N., \& Dunlop, J. 2007, MNRAS, 376, 151

Lacy, M., Petric, A. O., Sajina, A., et al. 2007, AJ, 133, 186

Lacy, M., Storrie-Lombardi, L. J., Sajina, A., et al., 2004, ApJS, 154, 166

La Franca, F., Fiore, F., Comastri, A., et al. 2005, ApJ, 635, 864

Lonsdale, C. J., Smith, H. E., Rowan-Robinson, M., et al. 2003, PASP, 115, 897

Lumb, D. H., Warwick, R. S., Page, M., \& De Luca, A. 2002, A\&A, 389, 93

Lutz, D., Maiolino, R., Spoon, H. W. W., \& Moorwood, A. F. M. 2004, A\&A, 418, 465

Mainieri, V., Rosati, P., Tozzi, P., et al. 2005, A\&A, 437, 805

Maiolino, R., Comastri, A., Gilli, R., et al., 2003, MNRAS, 344, 59

Maiolino, R., Mignoli, M., Pozzetti, L., et al. 2006, A\&A, 445, 457

Maiolino, R., Shemmer, O., Imanishi, M., et al. 2007, A\&A, 468, 979

Martínez-Sansigre, A., Rawlings, S., Lacy, M., et al., 2005, Nature, 436, 666

Martínez-Sansigre, A., Rawlings, S., Bonfield, D. G., et al. 2007, MNRAS, 379, L6

McMahon, R. G., Walton, N. A., Irwin, M. J., et al. 2001, New Astron. Rev., 45, 97

Menci, N., Fiore, F., Puccetti, S., \& Cavaliere, A. 2008, ApJ, 686, 219

Merritt, D., \& Ferrarese, L., 2001, The Central Kiloparsec of Starbursts and AGN: The La Palma Connection, ASP Conf. Proc., 249, 335

Norman, C., Hasinger, G., Giacconi, R., et al. 2002, ApJ, 571, 218

Page, M. J., Stevens, J. A., Ivison, R. J., \& Carrera, F. J. 2004, ApJ, 611, L85

Perola, G. C., Puccetti, S., Fiore, F., et al. 2004, A\&A, 421, 491

Piconcelli, E., Jimenez-Bailón, E., Guainazzi, et al. 2005, A\&A, 432, 15

Piconcelli, E., Fiore, F., Nicastro, et al. 2007a, A\&A, 473, 85

Piconcelli, E., Bianchi, S., Guainazzi, M., Fiore, F., \& Chiaberge, M. 2007b, A\&A, 466, 855

Polletta, M., Wilkes, B. J., Siana, B., et al., 2006, ApJ, 642, 673

Polletta, M., Tajer, M., Maraschi, L., et al. 2007, ApJ, 663, 81

Polletta, M., Weedman, D., Hönig, S., et al. 2008a, ApJ, 675, 960

Polletta, M., Omont, A., Berta, S., et al. 2008b, A\&A, 492, 81

Pope, A., Bussmann, R. S., Dey, A., et al. 2008a, ApJ, 689, 127

Pope, A., Chary, R. R., Alexander, D. M., et al. 2008b, ApJ, 675, 1171

Pozzi, F., Vignali, C., Comastri, A., et al. 2007, A\&A, 468, 603

Reeves, J. N., \& Turner, M. J. L. 2000, MNRAS, 316, 234

Reeves, J. N., Fabian, A. C., Kataoka, J., et al., 2006, AN, 327, 1079

Risaliti, G. 2002, A\&A, 386, 379

Rosati, P., Tozzi, P., Giacconi, R., et al. 2002, ApJ, 566, 667

Rowan-Robinson, M., Babbedge, T., Oliver, S., et al. 2008, MNRAS, 386, 697

Sajina, A., Lacy, M., \& Scott, D. 2005, ApJ, 621, 256

Sajina, A., Yan, L., \& Armus, L. 2007, ApJ, 664, 713

Seymour, N., Ogle, P., De Breuck, C., et al. 2008, ApJ, 681, L1

Sekiguchi, K., Akiyama, M., Furusawa, H., et al. 2005, Proceedings of the ESO

Workshop, Multiwavelength mapping of galaxy formation and evolution, ed. A. Renzini, \& R. Bender, 82

Silk, J., \& Rees, M. J. 1998, A\&A, 331, L1

Simpson, C. 2005, MNRAS, 360, 565

Somerville, R. S., Hopkins, P. F., Cox, T. J., Robertson, B. E., \& Hernquist, L. 2008, MNRAS, 391, 481

Steffen, A. T., Barger, A. J., Cowie, L. L., Mushotzky, R. F., \& Yang, Y. 2003, ApJ, 596, L23

Sturm, E., Hasinger, G., Lehmann, I., et al., 2006, ApJ, 642, 81

Surace, J. A., et al. 2004, BAAS, 36, 1450

Tozzi, P., Gilli, R., Mainieri, V., et al., 2006, A\&A, 451, 457

Turner, T. J., George, I. M., Nandra, K., \& Mushotzky, R. F. 1997, ApJS, 113, 23

Ueda, Y., Akiyama, M., Ohta, K., \& Miyaji, T. 2003, ApJ, 598, 886

Vignali et al. 2008, submitted

Weedman, D. W., Le Floc'h, E., Higdon, S. J. U., et al. 2006, ApJ, 638, 613

Worsley, M. A., Fabian, A. C., Bauer, F. E., et al. 2005, MNRAS, 357, 1281

Yan, L., Chary, R., Armus, L., et al., 2005, ApJ, 628, 604

Yan, L., Sajina, A., Fadda, D., et al., 2007, ApJ, 658, 778

Yun, M. S., Aretxaga, I., Ashby, M. L. N., et al. 2008, MNRAS, 389, 333 